\title{
Semiconductors for terahertz photonics applications
}

\author{
Arūnas Krotkus \\ Semiconductor Physics Institute, 01800, A. Goštauto 11, Vilnius, Lithuania
}

\begin{abstract}
:
Generation and measurement of ultrashort, sub-picosecond pulses of electromagnetic radiation with their characteristic Fourier spectra that reach far into terahertz $(\mathrm{THz})$ frequency range has recently become a versatile tool of the far-infrared spectroscopy and imaging. This technique - THz time-domain spectroscopy, in addition to a femtosecond pulse laser, requires semiconductor components manufactured from materials with a short photoexcited carrier lifetime, high carrier mobility, and large dark resistivity. Here we will review most important developments in the field of investigation of such materials. Main characteristics of low-temperature-grown or ion-implanted GaAs and semiconducting compounds sensitive in the wavelength ranges around $1 \mu \mathrm{m}$ and $1.5 \mu \mathrm{m}$ will be surveyed. The second part of the paper is devoted to the effect of surface emission of $\mathrm{THz}$ transients from semiconductors illuminated by femtosecond laser pulses. Main physical mechanisms leading to this emission as well as their manifestation in various crystals will be described.
\end{abstract}




\section{Introduction}

Terahertz (THz) frequency domain is usually defined as the portion of the submillimeterwavelength electromagnetic spectrum between approximately $1 \mathrm{~mm}(300 \mathrm{GHz})$ and $30 \mu \mathrm{m}(10$ $\mathrm{THz}$ ). The $\mathrm{THz}$ frequency range offers important technical advantages when compared with radio frequency electronics, such as wider bandwidth or improved spatial resolution, and promises new sensing applications (e.g., concealed weapon detection, chemical and biological agent detection, medical diagnostics, etc.). However, this range also presents significant challenges associated with strong atmospheric attenuation, weak interaction signatures, and, most importantly, fundamental engineering problems that until now have severely limited the implementation of conventional electronics technologies into this regime. Capability of solid-state electronics at $\mathrm{THz}$ frequencies has always been very limited, because both the power and efficiency of microwave devices significantly decrease when the active region length is scaled-down to sub-micrometer dimensions corresponding to the $\mathrm{THz}$ frequency range. At the opposite, high frequency side of this so-called "THz gap", the development of diode lasers is impeded by the fact that THz quantum energy is comparable with electron thermal energy, thus complicating the establishment of inverse population at room temperatures. Therefore, although there has been a considerable interest for the $\mathrm{THz}$ frequency range since the middle of the previous century, all previous high expectations have ended with disappointment.

Fortunately, recent important investigations have begun to define new approaches to generation and detection of $\mathrm{THz}$ waves. These approaches have triggered parallel development of commercially available $\mathrm{THz}$ systems, which already can be considered as enhanced and cost effective. This activity is surveyed in numerous, for such comparatively new field of research, editions [1-5].

The concept of THz time-domain spectroscopy (THz-TDS) is the first and the largest wave of recent research related to the $\mathrm{THz}$ frequency range techniques: in exploits $\mathrm{THz}$ wave generation and detection by semiconductor components activated by femtosecond laser pulses. After their advent in the mid-1960s [6], there has been a spectacularly rapid progress in the development of ultrafast lasers that has lasted for four decades (Fig. 1). The coupling between the ultrafast lasers and the semiconductor technology has led in mid-1970s to the birth of a new area of applied physics known as ultrafast optoelectronics. It started with the pioneering work of D. Auston [7], where an optoelectronic switch made of silicon was demonstrated. Photoexcited by a picosecond laser the switch could generate electrical pulses with picosecond rise and fall times. This work has initiated a 
wide field of research (see, e.g., [8]) that soon split into two main branches. The first group of ultrafast optoelectronic activities has been aiming at the achievement of high-voltage picosecond electrical pulses and was employing for the switches materials with high electron mobility, large breakdown field, but relatively long (of the order of hundreds of picoseconds) carrier lifetimes such as GaAs:Cr or InP:Fe. High-voltage pulses generated by optoelectronic switches were successfully used in jitter-free switching of streak cameras [9], for biasing electro-optic elements such as Pockels cells or Kerr cells in active pulse shaping systems for laser fusion experiments [10], as well as for measuring hot-electron characteristics of semiconductors [11].

Another category of ultrafast optoelectronic applications exploits its high speed - the most important characteristic of the device. With electrical pulse rise time approaching 1 ps, oscilloscopes and other electronic equipment became too slow, thus new high-speed electrical measurement techniques were needed. The solution was to use parts of the same laser pulse for two purposes: generating the electrical pulse and its sampling. Picosecond sampling of electrical transients by ultrafast photoconductors [12] and, later on, by using the electro-optic effect in a Pockels cell integrated with the transmission line along which these transients were propagating [13] were introduced in early 1980s. Low voltage of the generated electrical transients was in this case unavoidable consequence of the switch material selection - short carrier lifetimes in semiconductors are as a rule obtained at the cost of a significant reduction of their mobility. Amorphous silicon, from which the first optoelectronic switches were made for high-speed applications has extremely low electron mobility, therefore the amplitude of the switched electrical transients was very small, too.

With the advent of femtosecond lasers [14], optoelectronic devices have reached subpicosecond speeds. Most transmission lines, including micro-striplines that have been used in picosecond optoelectronics, are becoming very dispersive at this time scale. Important breakthrough was again achieved by D. Auston's group at AT\&T Bell Labs. They were the first to integrate picosecond photoconductors with wide-band dipole antennae for both generating and detecting ultrashort electrical transients [15] and to realize in such a way the free-space transmission of these transients. In this work, a new semiconductor material with subpicosecond recombination times ion-bombarded silicon-on-sapphire (SOS), which was found to have significantly higher electron mobility $\left(\sim 30 \mathrm{~cm}^{2} / \mathrm{Vs}\right)$ than amorphous $\mathrm{Si}$, was used for the first time.

The distance between emitter and detector in [15] was only $2 \mathrm{~mm}$, therefore it has been too early to speak about spectroscopic applications of such a system. The first THz-TDS system as we know it today ( Fig. 2) was developed by Ch. Fattinger and D. R. Grischkowsky, who also used dipole antennae manufactured from radiation damaged SOS [16, 17]. They have additionally 
introduced hemi-spherical surface lenses from crystalline sapphire and parabolic mirrors for collimating $\mathrm{THz}$ beams, thus increasing the propagation distance of those beams to $\sim 100 \mathrm{~cm}$. Newly developed THz-TDS systems had important advantages over traditional measurement techniques in this frequency range: extremely sensitive detection of the far-infrared radiation, larger than $10^{5}$ signal-to-noise ratio, and coherent detection of the electric field for simultaneous measurement of both the phase and the amplitude of different frequency components of the $\mathrm{THz}$ pulse. These advantages were used by the group from IBM for measuring $\mathrm{THz}$ spectra of water vapour [18] as well as of different dielectric and semiconducting materials [19].

Successive developments of THz-TDS systems have been highly dependent on the optimization of material characteristics crucial for the photoconductive switch performance: lifetime, electron mobility, dark resistance, and breakdown field. Selection of the switch material did also rely on the wavelength of the femtosecond laser that is used for switch photoexcitation. For colliding-pulse mode-locked $(\mathrm{CPM})$ dye lasers $(\lambda \approx 620 \mathrm{~nm})$, which were used in the first THz-TDS systems, the best material for photoconductive switches was radiation-damaged SOS layer. After Kerr-lens mode-locked Ti:sapphire laser $(\lambda \approx 800 \mathrm{~nm})$ has been commercially introduced and became the main tool for ultrafast measurements, most widely used material became GaAs grown by molecular-beam-epitaxy (MBE) at low substrate temperatures (LTG GaAs) [20]. Presently, this research is shifted to semiconductors with relatively narrow bandgap that could be used together with femtosecond lasers emitting at infrared wavelengths from $1 \mu \mathrm{m}$ to $1.55 \mu \mathrm{m}$.

$\mathrm{THz}$ radiation pulse could be generated even at unbiased semiconductor surfaces illuminated by a femtosecond laser beam. This phenomenon was discovered in early 1990-s [21]. Surface THz emission is caused by several different physical effects whose contribution varies from material to material. Investigations of $\mathrm{THz}$ surface emission, which were very intensive during the last decade [22], have not only led to the discovery of efficient $\mathrm{THz}$ radiation sources, but also became an important tool of material characterisation.

This paper is organized as follows. Section 2 of it will summarize main material parameters that are important for optoelectronic $\mathrm{THz}$ components. In Section 3 semiconductors used for manufacturing such components activated by Ti:sapphire laser and longer wavelength lasers will be described, and Section 4 will review the effects of surface emission of $\mathrm{THz}$ pulses from different semiconductor crystals.

\section{Performance characteristics of THz-TDS systems}

Electrically biased photoconductor generates a $\mathrm{THz}$ pulse when a laser pulse induces its conductivity changes. Transient photoconductivity is highly complex phenomenon, it includes 
optical generation of hot electrons and holes, their rapid thermalization, ballistic acceleration of the electrons, velocity-overshoot on a subpicosecond time scale [23], and fast screening of the internal electric field. The shape of the emitted $\mathrm{THz}$ pulse depends on design of antenna and on the conditions of its propagation in external quasi-optical set-up. This article primarily focuses on the characteristics of semiconductor materials used in the photoconductive $\mathrm{THz}$ components and only basic features of $\mathrm{THz}$ generation and detection will be discussed.

The THz radiation is generated in a photoconductor theoretically described by Maxwell's equations

$$
\begin{gathered}
\nabla \times \vec{E}(t)=\frac{\partial \vec{B}(t)}{\partial t} \\
\nabla \times \vec{B}(t)=\mu_{0} \vec{J}(t)+\epsilon_{0} \mu_{0} \frac{\partial \vec{E}(t)}{\partial t},
\end{gathered}
$$

where $E(t)$ is the electric field, $B(t)$ is the magnetic field, $J(t)$ is the total current density due to free and bond carrier contributions, and $\epsilon_{0}, \mu_{0}$ are the permittivity and the permeability of free space, respectively. Any physical process that causes fast changes in material properties after its excitation with a femtosecond laser pulse can act as a source of generated $\mathrm{THz}$ radiation. In photoconductors, the material parameter that is changed most is its electrical conductivity $\sigma$. Therefore, the $\mathrm{THz}$ electric field $E_{T H z}(t)$ radiated by traditional emitting antenna, which behaves as a Hertzian dipole [16], will be directly proportional to the first derivative of the current flowing in the photoexcited gap of the antenna $j_{e}$ :

$$
E_{T H z}(t) \propto \frac{d j_{e}(t)}{d t} .
$$

The discussion that follows will, therefore, be limited to the analysis of the dynamic photoconductance. We will use the analytical model of $\mathrm{THz}$ pulse generation and detection in photoconductive switches developed in [24]. A comprehensive study of the effects of quasi-optical THz-TDS systems can be found in [25]. Although the model used in [24] corresponds to a linear case, when the characteristic times of various carrier transport and recombination processes do not depend on the optical excitation level, the results of the model can provide important insights on how the parameters of semiconductor material affect the performance of THz-TDS system.

The first step in generating $\mathrm{THz}$ radiation is photoexcitation of the $\mathrm{DC}$ biased region of a semiconductor. It is important that the energy bandgap of the semiconductor $\varepsilon_{g}$ is smaller than the laser photon energy $\hbar v$, so that the photons will be absorbed and electron and hole pairs will be 
created. It is assumed in [24] that the non-equilibrium carriers created in the semiconductor by a Gaussian-shaped laser pulse with the duration of $\tau_{\text {las }}$ and decay single-exponentially with characteristic times of $\tau_{e m}$ - for the $\mathrm{THz}$ emitter and of $\tau_{\text {det }}$ - for the $\mathrm{THz}$ detector. Only the contribution of more mobile electrons is taken into account when the temporal evolution of the photocurrent is evaluated; the dynamics of the average photoelectron velocity $v(t)$ is described by the classical Drude model and the rate equation

$$
\frac{d v(t)}{d t}=\frac{q}{m} E(t)-\frac{v(t)}{\tau_{m e m, m \operatorname{det}}},
$$

where $q$ is the elementary charge, $m$ is the electron effective mass, and $\tau_{m e m}, \tau_{m \text { det }}$ are the momentum relaxation times for electrons in the emitter and the detector materials, respectively.

After the convolution of the time dependences of the optical pulse intensity, the electron density, and their drift velocity, one obtains the expression of the photocurrent density in the emitter [24]:

$$
\begin{aligned}
& j_{\text {em }}(t)=\left\{\exp \left(\frac{\tilde{\tau}_{\text {las }}^{2}}{4 \tau_{\text {em }}^{2}}-\frac{t}{\tau_{\text {em }}}\right) \operatorname{erfc}\left(\frac{\tilde{\tau}_{\text {las }}}{2 \tau_{\text {em }}}-\frac{t}{\tilde{\tau}_{\text {las }}}\right)-\exp \left(\frac{\tau_{\text {las }}^{2}}{4 \tilde{\tau}_{\text {em }}^{2}}-\frac{t}{\tilde{\tau}_{\text {em }}}\right) \operatorname{erfc}\left(\frac{\tilde{\tau}_{\text {las }}}{2 \tilde{\tau}_{\text {em }}}-\frac{t}{\tilde{\tau}_{\text {las }}}\right)\right\} \times \\
& \times \frac{P_{e m} E_{d c} \tau_{m e m}}{m}
\end{aligned}
$$

where $\tilde{\tau}_{\text {las }}=\tau_{\text {las }} / 2 \sqrt{\ln 2}$ and $1 / \widetilde{\tau}_{e m}=1 / \tau_{\text {em }}+1 / \tau_{\text {mem }}, P_{e m}$ is the absorbed laser power, and $E_{d c}$ is the electric field in the emitter.

Fig. 3 shows the transient photocurrent in the emitter and the radiated $\mathrm{THz}$ pulse in the far field, calculated from (4) and (3), respectively, together with the temporal shape of the excitation pulse. These transients correspond to two different emitters made from materials with carrier lifetimes $\tau_{e m}$ of 200 fs and 2 ps. The electron momentum relaxation times for these two materials were corresponding to the parameters of LTG GaAs ( $\tau_{\text {mem }}=20 \mathrm{fs}$ ) and semi-insulating (SI) GaAs ( $\tau_{\text {mem }}=200 \mathrm{fs}$ ). The emitter with a longer lifetime produces an almost unipolar waveform, whereas the waveform generated by the emitter made from a short-lifetime material is bipolar. In both cases the electromagnetic pulses have durations less than 1 ps because a step-like photocurrent produces delta-like pulse in the far field. The duration of the radiated pulse is limited by the rise time of the induced photocurrent, which is approaching the laser pulse duration; therefore, the $\mathrm{THz}$ pulses can be radiated also from the switches made from semiconductors with long carrier lifetimes. For the 
first time such a possibility has been demonstrated experimentally in [26]. Carrier lifetime cannot, however, be longer than the period between subsequent laser pulses, during which the resistance of the photoconductor and the electric field inside recover. This condition is fulfilled for typical femtosecond laser pulse repetition rates of several tens of MHzs in compensated semi-insulating semiconductor crystals with carrier lifetimes shorter than $1 \mathrm{~ns}$.

$\mathrm{THz}$ pulse detection with photoconductively gated antennae is based on similar physical principles as the $\mathrm{THz}$ pulse generation. For detecting $\mathrm{THz}$ pulses with subpicosecond temporal resolution, a dipole antenna is fabricated on a semiconductor with a short carrier lifetime and is excited by ultrashort laser pulse for rapid change in the conductivity. The photoconductive gap between the contacts of the detector antenna is, in this case, biased by the THz pulse. The electrical current in the detector circuit will be proportional to the amplitude of $\mathrm{THz}$ pulse during the detector excitation. Because both the emitter and the detector are activated by two parts of the same laser pulse the system is jitter-free.

Fig. 4 shows the temporal dependence of the photocurrent in the detector calculated after Eq.(12) from Reference 24. The emitter material parameters were corresponding to SI-GaAs; for the detector material parameters - to SI-GaAs and LTG GaAs. The shape of the incoming THz pulse is also shown for comparison. For both detectors the sampled photocurrent has a sharp step in the middle and smoothly varying wings that are caused by the photoexcited electron density relaxation in the detector material. It is important to note that the sharp photocurrent step (corresponding to the $\mathrm{THz}$ pulse arrival at the detector simultaneously with its illumination by the laser pulse) is present also when the device with a slower carrier recombination is used. Possibility of using long lifetime photoconductors for $\mathrm{THz}$ pulse sampling was, for the first time, demonstrated in [27], where subpicosecond electric transients were sampled with a photoconductor made from GaAs wafer with 1 ns long carrier lifetime. Kono et.al. [28] have demonstrated frequency bandwidth of $40 \mathrm{THz}$ for a THz-TDS system with a photoconductive detector manufactured from LTG GaAs and activated by 15 fs duration laser pulses. The bandwidth is much larger than it could be expected from the carrier lifetime of 1.4 ps of this material.

In the case of such ultra-broadband detection, the photoconductive antenna works as an integrating detector, thus the $\mathrm{THz}$ waveform is proportional to the derivative of the measured photocurrent [27, 29]. In [29] the system used in Ref. [28] was supplemented with a mechanical shaker for the $\mathrm{THz}$ pulse differentiation. This has enhanced the detection efficiency of the photoconductive antenna and has extended its detectable bandwidth to $60 \mathrm{THz}$. 
The spectral dependence of the detector current $j_{\text {det }}(f)$ can be obtained by applying the Fourier transform to the expression for $j_{\text {det }}(t)[24]$ :

$$
j_{\mathrm{det}}(f) \propto \frac{P_{e m} P_{\mathrm{det}} E_{d c} \tau_{e m} \tau_{\mathrm{det}} \tilde{\tau}_{e m} \tilde{\tau}_{\mathrm{det}} f \exp \left(-2\left(\pi f \tilde{\tau}_{l a s}\right)^{2}\right)}{m^{2}\left(1-2 i \pi f \tau_{e m}\right)\left(1+2 i \pi f \tau_{\mathrm{det}}\right)\left(1-2 i \pi f \tilde{\tau}_{e m}\right)} .
$$

Fig. 5 compares Fourier spectra of the transients measured by two different TDS systems. Here, as it has been done before, both photoconductive $\mathrm{THz}$ components are supposedly made of the same semiconductor with different electron mobilities and lifetimes. The comparison of these two spectra shows that the spectrum roll-off is much steeper for the TDS system with devices made of a longer lifetime material, therefore its frequency bandwidth for the same signal-to-noise ratio $(\mathrm{S} / \mathrm{N}=50 \mathrm{~dB})$ will be almost twice narrower. Although the main source of the noise in THz-TDS systems is the laser power fluctuations, the long-lifetime material with a larger average photocurrent can lead to a significant growth of the thermal noise, and this bandwidth difference can be even larger.

It should be pointed out that equation (3) for the description of photoexcited electron dynamics is approximate and introduces significant errors in estimated performance of the system. Typical momentum relaxation times in pure semiconductors are of the order of $100 \mathrm{fs}$, and this parameter sets a limit to the ultimate frequencies radiated by the emitter when laser pulses shorter than $\tau_{\text {mem }}$ are used for its excitation. However, one has to remember that the electric fields in the emitter are usually strong, therefore, the electron velocity overshoot effect [30] causes a faster rise of the photocurrent and higher radiated frequencies. Transient electron drift velocity overshoot effect is especially pronounced in GaAs, where the inter-valley transfer leads to a sharp reduction in $\tau_{\text {mem }}$ at high energies. The time scale $\Delta t$ when the electric field $\mathrm{E}$ accelerates the electrons electric ballisticaly and they move with a larger than the steady state velocity before reaching energy $\Delta \varepsilon$ necessary for the inter-valley transfer equals to

$$
\Delta t=\frac{\sqrt{2 m \Delta \varepsilon}}{q E} .
$$

For typical electric fields in GaAs photoconductive THz emitters of the order of $100 \mathrm{kV} / \mathrm{cm}$ and $\Gamma$-X inter-valley separation of $\Delta \varepsilon=0.42 \mathrm{eV}$, one obtains that the electrons are accelerated during $\sim 60 \mathrm{fs}$ and are afterwards sharply decelerating due to the scattering to subsidiary, high effective mass $\mathrm{X}$ valleys. Therefore the photocurrent rise time and, consequently, the radiated $\mathrm{THz}$ pulse duration will be much shorter that $\tau_{m e m}$. This effect has been demonstrated experimentally in [31]. 
For a $\mathrm{THz}$ detector made of a material with a subpicosecond carrier lifetime, the electrons photoexcited with a significant excess energy can be trapped even before reaching the thermal equilibrium with the crystalline lattice. Because they will remain hot during their whole presence in the conduction band, the ionized-impurity scattering will be much weaker than that at the thermal equilibrium (the scattering rate for this mechanism decreases with increasing energy as $\mathcal{E}^{-3 / 2}$ ); this will result in an enhanced mobility [32] and larger detector sensitivity. In conclusion, for both $\mathrm{THz}$ pulse emitters and detectors semiconductors with short electron lifetimes are preferable; this requirement is more stringent for the optoelectronic $\mathrm{THz}$ detectors.

\section{Semiconductors with ultrafast carrier recombination}

\subsection{Radiation-damaged silicon-on-sapphire}

The search for the semiconductor materials with ultrafast carrier recombination has begun in early 1980-s when the need for faster sampling gates for picosecond optoelectronic systems became evident. The first material was amorphous silicon (a-Si) [33]. Samples of a-Si prepared by three different methods have been investigated in [33]; the shortest carrier lifetime (less than 4 ps) was found in material prepared by thermal evaporation technique. Temporal resolution of the electronic correlation measurement used in this work was limited by the laser pulse duration and the circuit response times; it has been demonstrated later in experiments performed with sub-picosecond resolution [34] that the carrier lifetimes in a-Si can be as short as $\sim 800$ fs. However, the main disadvantage of a-Si as a substrate for photoconductive $\mathrm{THz}$ range components is very small its electron mobility, typically below few $\mathrm{cm}^{2} / \mathrm{Vs}$ for the material with sub-picosecond carrier lifetimes.

Later on, the main attention became focused on another variety of Si layers - radiation damaged silicon grown on sapphire substrates. It has been found in [35] that free-carrier trapping rate in this material increases linearly with $\mathrm{O}^{+}$-ion implantation dose and the measured carrier lifetime decreases down to the limit of $600 \mathrm{fs}$ at doses above $3 \cdot 10^{14} \mathrm{~cm}^{-2}$. Fig. 6 shows the carrier lifetime in SOS as a function of the implanted $\mathrm{O}^{+}$-ion dose. As a most probable cause of this carrier lifetime limit, the authors of [35] pointed out the amorphisation of the crystalline silicon and the saturation of the trap density. Similar conclusion was made in [36], where transient carrier dynamics in the radiation-damaged SOS was measured by the optical pump-THz probe technique. Average carrier mobilities were also evaluated in [36]; they were changing from $383 \mathrm{~cm}^{2} / \mathrm{Vs}$ in a sample with the lifetime of $5.5 \mathrm{ps}$ to $4.4 \mathrm{~cm}^{2} / \mathrm{Vs}$ in a-Si sample with the lifetime of $0.6 \mathrm{ps}$.

Radiation damaged SOS was the material for the first Hertzian dipoles radiating THz pulses into the free space [15] and for the components of the first THz-TDS system [16]. The 
photoconducting SOS antennae were used in almost all pulsed $\mathrm{THz}$ systems activated by femtosecond CPM dye lasers (quantum energy of $\sim 2 \mathrm{eV}$ ) [37]. Only when a new femtosecond laser system - Kerr-lens mode-locked Ti:sapphire laser - was introduced [38], another semiconductor low-temperature-grown GaAs - became the main material for the photoconductive $\mathrm{THz}$ range components.

\subsection{Low-temperature-grown GaAs}

\subsubsection{Structure and electrical characteristics.}

Normally, GaAs layers are grown by MBE technique at temperatures of $550^{\circ} \mathrm{C}-650^{\circ} \mathrm{C}$. In 1988, Smith et.al. successfully used GaAs grown at $200^{\circ} \mathrm{C}$ as a high-resistivity buffer layer in the fabrication of GaAs FET's [39]. Shortly thereafter, photodetectors made from GaAs grown at $200^{\circ} \mathrm{C}$ and annealed at $600^{\circ} \mathrm{C}$ were shown to have switching speeds of $\sim 500 \mathrm{fs}$ and excellent sensitivity [40]. Since then LTG GaAs became the most important material for applications in ultrafast optoelectronics [41]. Indeed, LTG GaAs and related materials match most of the requirements for making efficient ultrafast optoelectronic devices such as short carrier lifetime, high electron mobility, high electric breakdown field, and large dark resistivity [42]. Components manufactured from LTG GaAs have been used for subpicosecond electric pulse generation [43], all optical switching [44], laser mode locking [45], and, most importantly, for generation and detection of $\mathrm{THz}$ pulses with extremely broad frequency bands $[46,47]$ as well as for narrow-band $\mathrm{CW} \mathrm{THz}$ generation [48].

Unlike conventional MBE grown GaAs, GaAs layers grown at $200-300^{\circ} \mathrm{C}$ are nonstoichiometric with up to $1 \%$ of excess As. The structural defects caused by the non-stoichiometry are controlling the majority of physical characteristics of this material. In as-grown LTG GaAs, the excess arsenic is taken up by point defects, most common of which are the arsenic-antisite defects $\mathrm{As}_{\mathrm{Ga}}$, arsenic interstitials $\mathrm{As}_{\mathrm{i}}$, and gallium vacancies $\mathrm{V}_{\mathrm{Ga}}$. Complex study of LTG GaAs layers by various structural, electrical, and optical investigation techniques performed in [49] has led the authors of this work to a conclusion that As antisite related defects containing two arsenic atoms can account for all the As in access in this material. A high concentration of $\mathrm{As}_{\mathrm{Ga}}$ (up to $10^{19} \mathrm{~cm}^{-3}$ in layers grown at $200^{\circ} \mathrm{C}$ ) has been observed in the material from near infrared absorption and magnetic circular dichroism of absorption [50]; these defects exhibit EL2-defect like photoquenching behaviour [51]. At this growth temperature, $\mathrm{V}_{\mathrm{Ga}}$ density is lower by approximately one order of magnitude [52], but their presence is extremely important for the compensation of $\mathrm{As}_{\mathrm{Ga}}$ as well as for the impurity diffusion processes. 
Numerous $\mathrm{As}_{\mathrm{Ga}}$ defects form a defect band in as-grown layer, therefore hopping conduction is dominating [53] with the resistivity in the range of $10-10^{3} \Omega \cdot \mathrm{cm}$. The resistivity of LTG GaAs increases to $10^{6}-10^{7} \Omega \cdot \mathrm{cm}$ after post-growth annealing at temperatures higher than $600^{\circ} \mathrm{C}$ [54]. The annealing also leads to a significant improvement in the crystallographic quality of the layers [55] and to the formation of spherical metallic As precipitates [56] and radii distribution depending on the annealing temperature (Fig. 7). Excess arsenic precipitation causes significant reduction $\mathrm{As}_{\mathrm{Ga}}$ defect density down to approximately $10^{16}-10^{17} \mathrm{~cm}^{-3}$ [50], Fig. 8 shows annealing temperature dependences of the extended arsenic defect density and their size [55-57].

High density of excess arsenic related defects is believed to be responsible also for high breakdown fields of LTG GaAs [58]. It has been found that in as-grown at $200^{\circ} \mathrm{C}$ layer this field can be as high as $320 \mathrm{kV} / \mathrm{cm}$, it slightly decreases after annealing (to $250 \mathrm{kV} / \mathrm{cm}$ at the annealing temperature of $650^{\circ} \mathrm{C}$ ), but still remains more than one order magnitude higher than in SI-GaAs [58]. It was concluded in [58] that enhanced breakdown fields in LTG GaAs are caused by two main effects. First, the LTG GaAs layer passivates the SI-GaAs substrate and eliminates the surface breakdown. Second, a higher hoping conduction minimizes accumulation of injected charge in the cathode region, and the resultant more uniform distribution of the electric field prevents the premature breakdown either at the anode-contact region or at the surface.

\subsubsection{Electron trapping times}

Electron trapping times in LTG GaAs are usually much shorter than $1 \mathrm{ps}$, thus they are far too short to be measured by purely electronic techniques. Instead, various pump-probe type experiments with ultrashort laser pulses are the main tools for accessing the ultrafast carrier density relaxation in semiconductors. A part of a pulsed laser beam induces the change in a certain physical characteristic of the sample, and this change is monitored with a second part of the same beam that arrives at the sample at different time delays. Carrier dynamics was documented by pump- probe measurements of several different physical characteristics of LTG GaAs: photocurrent transients [59], dynamic reflectance [60], dynamic transmittance [61], time-resolved photoluminescence [62], and $\mathrm{THz}$ conductivity [63]. All these physical characteristics are mainly sensitive to the presence of the non-equilibrium electrons in the sample; therefore, the electron dynamics in LTG GaAs is fairly well understood.

Energy distribution of non-equilibrium electrons immediately after their photo-excitation with an ultrashort laser pulse corresponds to the laser pulse spectrum, and the electron and hole distributions remain inverted for some time. Subsequently, the distributions approach their 
thermalized shapes, while the electron-hole plasma cools down by interacting with the phonons [64, 65]. These processes proceed at rates dependent on the electron excess energy (i.e., on the laser wavelength) and on the excitation level. Characteristic times of the photo-excited electron thermalization are several picoseconds at low excitation levels and decrease down to $\sim 100$ fs for photo-excited carrier densities of the order of $10^{18} \mathrm{~cm}^{-3}$ [64].

In annealed LTG GaAs, where electron trapping times are longer than 200 fs, the photoexcited electron density dynamics can be characterised by the majority of the pump-and-probe techniques. Fig. 9 shows the results of three different pump-and-probe measurements performed on a sample grown at $250^{\circ} \mathrm{C}$ and annealed at $650^{\circ} \mathrm{C}$. These data were obtained using femtosecond Ti:sapphire laser based experimental set-ups; all three transients show the characteristic decay time of $\sim 500 \mathrm{fs}$ that can be interpreted as the electron trapping time. The electron trapping time ranges between 0.2 ps and 1 ps in the annealed LTG GaAs layers; it can be controlled by changing As and $\mathrm{Ga}$ beam equivalent pressure ratio [66], the growth and anneal temperatures [67, 68], or by additionally doping the layer with acceptor impurity Be during the MBE growth [69].

Photo-excited electrons in as-grown LTG GaAs layers are trapped too fast to be characterised with standard femtosecond pump-and-probe techniques. In [70] the electron trapping time in asgrown LTG GaAs was determined indirectly, by investigating the photoluminescence (PL) spectra. As the electrons are trapped faster than they become thermalized, the PL spectrum is peaked not in the vicinity of the band-gap energy, but around the photo-excitation energy. The PL spectrum of asgrown at $250^{\circ} \mathrm{C} \mathrm{GaAs} \mathrm{layer} \mathrm{excited} \mathrm{by} \mathrm{femtosecond} \mathrm{Ti:sapphire} \mathrm{laser} \mathrm{pulses} \mathrm{is} \mathrm{shown} \mathrm{on} \mathrm{Fig.} 10 \mathrm{~b}$. It can be seen that a substantial signal is detected even at photon energies larger than the exciting laser quantum $(h v=1.6 \mathrm{eV})$, in contrast with the PL spectrum of annealed LTG GaAs that peaks at the energy close to the bandgap and is essentially the same as in the material grown at normal conditions. PL intensity of as-grown layer is much lower than that of annealed at $600^{\circ} \mathrm{C} \mathrm{LTG} \mathrm{GaAs}$ measured under similar experimental conditions. This decrease of the integral photoluminescence intensity yield allows the rough estimation of carrier lifetime in the as-grown sample. By interpolating the missing parts of the spectra and by assuming that the matrix elements for the radiative recombination for the as-grown and the annealed samples are the same, the carrier lifetime for as-grown LTG GaAs of about $60 \mathrm{fs}$ has been deduced [70]. Similar result ( $\tau_{l} \sim 70 \mathrm{fs}$ ) has been obtained also after a quantitative comparison of the measured PL spectrum with the results of the numerical simulation by a combined Monte Carlo / molecular dynamics procedure [70].

Figure 11 shows a collection of electron lifetimes in the LTG GaAs grown at different temperatures and annealed at $600^{\circ} \mathrm{C}[61,65,70-72]$. The available experimental data for the as- 
grown LTG GaAs [61, 70] are also presented for comparison. Despite some scatter of the experimental points (caused by slightly different technological conditions and difficulties in exact measurement of the substrate temperature around $200^{\circ} \mathrm{C}$ ), it is clear that the electron lifetimes in the as-grown LTG GaAs are always shorter than in the annealed layers. Such a comparison is an important proof for the point defect model of the carrier recombination in the LTG GaAs because the As-precipitates (present only in the annealed layers) cannot account for the observed ultrafast carrier dynamics in the as-grown LTG GaAs. Moreover, it has been argued in [73] that even in the annealed layers, where both As-precipitates and As-antisites are present, the electron trapping crosssection of the latter is larger than that of the former.

Another proof of the point defect model was obtained by investigating the LTG GaAs layers doped with Be impurities $[69,74]$. This model assumes that the non-equilibrium electrons in the LTG GaAs are trapped at the ionised $\mathrm{As}_{\mathrm{Ga}}$ donors. Because the total number of the $\mathrm{As}_{\mathrm{Ga}}$ defects is much larger than that of the main compensating acceptor $-\mathrm{V}_{\mathrm{Ga}}$, only a few percent of the Asantisites are ionised and can participate in the electron trapping. Neutral $\mathrm{As}_{\mathrm{Ga}}$ centres can be activated by doping with acceptor impurities, c.a., Be or C [75], for a fine adjustment of the free electron lifetime $[69,76]$.

\subsubsection{Hole trapping times}

The majority of the pump-and-probe experiments are sensitive to the electron density relaxation, because they are monitoring either conduction band filling effects (as in the transient optical reflectance and transmittance measurements), electrical conductivity (photocurrent or $\mathrm{THz}$ probe transients that are dominated by more mobile carriers), or, simply, the decay of the carriers that are trapped first (as in time-resolved PL measurements). Hole trapping times were usually evaluated from indirect experimental evidence. In particular, the shape of various pump-probe transients depends on holes at high photo-excitation intensities when the electron traps become saturated [77]. Also, the dynamics of the electron trap population can be monitored by the nearinfrared probe pulses generated by an optical parametric oscillator [78]. Direct measurement of the hole density dynamics in the valence band of LTG GaAs was performed in [79] by an original twocolour pump-and-probe-technique. In this experiment, the non-equilibrium electrons and holes were excited by femtosecond optical pulses at the wavelength of $800 \mathrm{~nm}$ and the inter-valence band transitions were probed with 9- $\mu \mathrm{m}$ wavelength mid-infrared (MIR) pulses. The latter wavelength corresponds to the resonance transitions between the heavy and the light hole bands as it is shown 
on Fig. 12. An important advantage of this scheme is that the inter-valence-band absorption at this wavelength is an one order of magnitude stronger than the free electron absorption [80].

The time-resolved measurements of the induced MIR absorption performed with this technique on the undoped and Si- or Be-doped as-grown LTG GaAs layers are shown in Fig.13 [79]. The hole trapping time in the as-grown, undoped material was close to $2 \mathrm{ps,}$, it decreased to less than 1 ps after Si-doping, and increased to 10 ps in Be-doped as-grown layer. Sample annealing slows done the dynamics by approximately 10 times. Because all this experimentally observed variation of the hole trapping times correlates with the changes of the neutral As-antisite density in the LTG GaAs, it has been concluded in [79] that these defects are the main hole traps in this material.

\subsection{Ion-implanted GaAs}

GaAs implanted with heavy, high energy ions of As was proposed as an alternative material for ultrafast semiconductor components to LTG GaAs in $[81,82]$. Because the characteristics of both materials are strikingly similar, ion-implanted GaAs could be used as a substrate material for photoconductive antennae or other components operating in the $\mathrm{THz}$ frequency range. The use of ion-implanted GaAs has several advantages over LTG GaAs. Ion dosage and hence the properties of the implanted material can be precisely and reproducibly tailored, whereas the growth temperature - the most important parameter of the LTG GaAs technology - in the MBE chamber is difficult to control in the actual growth temperature range $\left(200-300^{\circ} \mathrm{C}\right)$. Furthermore, ion implantation offers the feasibility of fine tuning the properties of the semiconductor by varying the appropriate implantation (and annealing) conditions. As yet, there are only few examples of ionimplanted GaAs applications in ultrafast optoelectronic devices, although the feasibility of such devices as semiconductor saturable absorber mirrors (SESAM) [83], photoconductive antennae [84], and surface field THz emitters [85] manufactured from ion-implanted GaAs has been already demonstrated in the literature.

Arsenic implanted and subsequently annealed at $600^{\circ} \mathrm{C} \mathrm{GaAs}$ has been reported to contain Asprecipitates and high resistivity [86]. It has been demonstrated later that the carrier recombination characteristics of the As-ion implanted GaAs closely remind those of LTG GaAs. E.g., electron trapping time in GaAs implanted by $2 \mathrm{MeV}$ energy ions was equal to $30 \mathrm{fs}$ before annealing [70] and 1 ps after annealing at the temperature of $600^{\circ} \mathrm{C}$ [81]. Spatially resolved carrier lifetime measurements of the As-ion implanted and annealed GaAs [87] have shown that carriers decay faster at the surface, whereas the precipitates are predominantly nucleating deeper in the bulk of the crystal, in the proximity of the ion-stop range. This observation evidences that point defects rather 
than precipitates are responsible for the ultrafast carrier recombination. Moreover, the annealing dynamics of the implanted GaAs closely reminds that of LTG GaAs. Arrhenius plot of the carrier trapping rate as a function of the annealing temperature (Fig.14) corresponds to the activation energy of $\sim 1.3 \mathrm{eV}$, which is very close to $1.4 \mathrm{eV}$ determined in [50] as the activation energy for the processes leading to the disappearance of $\mathrm{As}_{\mathrm{Ga}}-$ related defects in LTG GaAs.

Surprisingly enough, similar results were obtained also after implanting GaAs by other heavy ions, such as $\mathrm{Ga}, \mathrm{Si}$, and $\mathrm{O}$ [88]. The trapping time dependences on the implantation dose for these four implant species measured after $600^{\circ} \mathrm{C}$ annealing are shown on Fig. 15. In all cases, a monotonic decrease of the trapping time with the implantation dose was observed. Later on, similar results were also obtained for implanted with Ar ions and annealed GaAs [89]. In contrast to the heavy ion implantation, the implantation of GaAs with lighter ions such as $\mathrm{N}^{+}$[90] or $\mathrm{H}^{+}$[91] has resulted in short carrier lifetimes only for non-annealed material.

The mechanism leading to the stoichiometric disturbance in GaAs irradiated with heavy and energetic ions was considered in $[92,93]$. Due to the difference in $\mathrm{Ga}$ and As masses, the gallium atoms are recoiled after the ion implantation further into the bulk of the crystal, resulting in the surface layer of ion-implanted GaAs that is slightly As-rich, while the deeper region is slightly Garich. Therefore, the presence of excess arsenic, which could be the source of the $\mathrm{As}_{\mathrm{Ga}}$ related point defects, can be expected at the surface of all samples irradiated with heavy, high-energy ions. Such defects have been detected by electron-paramagnetic-resonance measurements in As-implanted samples [81] and in some of the $\mathrm{Ga}^{+}$-implanted samples [82].

The results shown on Fig. 15 were obtained from time-resolved PL transients. As the photoluminescence signal is proportional to the product of the electron and hole concentrations, its decay time corresponds to the lifetime of the carriers that are trapped first. In [94], the trapping times of non-equilibrium carriers in LTG GaAs as well as in GaAs crystals implanted by various ions were determined separately by using optical pump - THz probe technique for monitoring the electron density and optical pump - MIR probe experiment for measuring the hole trapping times. The results of this investigation are summarized on the diagram presented in Fig. 16; they can be used for discussing the prospects of application of non-stoichiometric GaAs in various optoelectronic $\mathrm{THz}$ range devices.

Femtosecond laser activated $\mathrm{THz}$ emitters and detectors are, essentially, photoconductive components integrated with wide band antennae. The $\mathrm{Si}^{+}$-implanted GaAs can be excluded from the consideration because of its large dark conductivity and rather long electron trapping times. On the other hand, $\mathrm{As}^{+}, \mathrm{Ga}^{+}$, and $\mathrm{O}^{+}$-implanted $\mathrm{GaAs}$ are highly-resistive after thermal annealing and can be used as substrates for $\mathrm{THz}$ photoconductors. The electron trapping times in these crystals are 
quite short; however, they approach 1 ps only when the ion-implantation dose is very large $\left(\geq 10^{16}\right.$ $\mathrm{cm}^{-2}$ ). For such large doses, the electron mobility in the material becomes small, thus a wide band of the devices is achievable only at the cost of their sensitivity. From this point of view the use of LTG GaAs is preferable, because the electron mobility remains at a satisfactory level even when their trapping times are much shorter than 1 ps in this material. From all ion-implanted materials investigated in [93] only $\mathrm{O}^{+}$-implanted GaAs can provide the electron trapping time and mobility product comparable with that for LTG GaAs. Moreover, $\mathrm{O}^{+}$-implanted GaAs crystals are, in general, characterized by considerably shorter hole trapping times than LTG GaAs. Short hole trapping times are essential for devices such as $\mathrm{CW} \mathrm{THz} \mathrm{emitters} \mathrm{based} \mathrm{on} \mathrm{the} \mathrm{optical} \mathrm{mixing} \mathrm{effect}$ [95] and this advantage of ion-implanted GaAs for the optical mixer fabrication has already been verified [96].

\subsection{Materials sensitive at $1 \mu \mathrm{m}$ and longer wavelengths}

\subsubsection{InGaAs}

Majority of the optoelectronic THz-TDS systems are presently using femtosecond Ti:sapphire lasers emitting at wavelengths around $800 \mathrm{~nm}$ and photoconductive components made from LTG GaAs epitaxial layers. Because Ti:sapphire laser requires many-stage optical pumping arrangement, these systems are bulky and complicated. Optoelectronic applications, including those for $\mathrm{THz}$ radiation devices, often require components operating at telecommunications wavelengths of 1.3 $\mu \mathrm{m}$ or $1.55 \mu \mathrm{m}$, because mature telecom technology is one of the most promising ways towards compact and low-cost terahertz systems. Femtosecond lasers emitting in the spectral range close to $1 \mu \mathrm{m}$ would be also advantageous. These lasers can be directly pumped by laser diode bars, too. Moreover, the requirements of cost, robustness, and flexibility for many $\mathrm{THz}$ applications the laser are compatible with fibre-optical cables. At the operation wavelength of $1 \mu \mathrm{m}$, the attenuation in the fibres is still reduced when compared to $800 \mathrm{~nm}$, and compact, $\mathrm{Nd}^{+}$or $\mathrm{Yb}^{+}$ion based, diodepumped solid-state or fibre lasers are available. However, the LTG GaAs is not an efficient photoconductor for excitation at these wavelengths $(1 \mu \mathrm{m}, 1.3 \mu \mathrm{m}$, and $1.55 \mu \mathrm{m})$, because its energy bandgap is larger than corresponding photon energy quanta and two-photon absorption processes are not efficient enough [97].

The unique properties of LTG GaAs are attributed to the As-rich conditions of this material; therefore, similar properties could be expected also in other As-containing III-V compounds. An interesting material for $\mathrm{THz}$ photoconductor applications is $\mathrm{In}_{\mathrm{x}} \mathrm{Ga}_{1-\mathrm{x}} \mathrm{As}$. This material has a lower bandgap than GaAs and its absorption edge can be tailored around the wavelengths of 1-1.55 $\mu \mathrm{m}$. 
Therefore, LTG $\operatorname{In}_{\mathrm{x}} \mathrm{Ga}_{1-\mathrm{x}}$ As can potentially offer advantages for photoconducting THz emitters and detectors, if high resistivity, short carrier lifetime, and high sensitivity of this material could be demonstrated. First attempts of low-temperature growth of $\operatorname{In}_{0.53} \mathrm{Ga}_{0.47} \mathrm{As}$ lattice matched to InP substrates were performed in [98]. It has been found that the layers grown at the lowest temperatures $\left(180^{\circ} \mathrm{C}\right)$ were characterised by rather short carrier lifetimes of $\sim 2.3 \mathrm{ps}$, but had very low resistivity. Doping of LTG $\operatorname{In}_{0.53} \mathrm{Ga}_{0.47}$ As with Be has led to some reduction of the free electron density $[99,100]$ (from approximately $2.4 \cdot 10^{18} \mathrm{~cm}^{-3}$ to $5 \cdot 10^{16} \mathrm{~cm}^{-3}$ (still too large for applications of this material in photoconductor devices); the carrier lifetime has decreased simultaneously to $\sim 1.9$ ps [101].

Ion-implantation was also employed for reducing carrier lifetimes in InGaAs layers by several groups. In [102] the $\operatorname{In}_{0.53} \mathrm{Ga}_{0.47} \mathrm{As}$ epitaxial layer with a residual electron concentration of $\sim 2 \cdot 10^{15}$ $\mathrm{cm}^{-3}$ was implanted by $\mathrm{Au}^{+}$ions with the energy of $200 \mathrm{keV}$. This has led to the reduction of the carrier lifetime by more than three orders of magnitude, to less than $1 \mathrm{ps}$. Although dark resistivity of the layer was rather low $(\sim 0.6 \Omega \cdot \mathrm{cm})$, photoconductive switches with the response times of $2.2 \mathrm{ps}$ were successfully manufactured from this material. Slightly better results (the lifetime of $\sim 0.5 \mathrm{ps}$ and the resistivity of $1.9 \Omega \cdot \mathrm{cm}$ ) were obtained later by the same group after implanting InGaAs with very high energy $(11 \mathrm{MeV}) \mathrm{Br}^{+}$ions [103]; this material was used to fabricate a continuous-wave $\mathrm{THz}$ emitter generating at frequencies up to $0.8 \mathrm{THz}$ by mixing the radiation from two laser diodes with the wavelengths around $1.55 \mu \mathrm{m}$. Combination of short carrier trapping times and large resistivities appropriate for ultrafast optoelectronic applications was so far achieved in [104] by implanting $\mathrm{In}_{0.53} \mathrm{Ga}_{0.47} \mathrm{As}$ with $\mathrm{Fe}^{+}$ions over a certain range of the implantation doses. It was found in [105] that a jump in sheet resistance to a value of $4 \cdot 10^{5} \Omega$ /square occurs for samples implanted at room temperature to a dose of $10^{15} \mathrm{~cm}^{-2}$ and annealed at $500^{\circ} \mathrm{C}$.

Another approach of obtaining materials for $\mathrm{THz}$ photoconducting devices sensitive to longwavelength laser radiation uses lattice-mismatched growth of $\operatorname{In}_{\mathrm{x}} \mathrm{Ga}_{1-\mathrm{x}} \mathrm{As}$ on $\mathrm{GaAs}$ substrates $\left(\mathrm{x}=0.25, \varepsilon_{\mathrm{g}}=1.18 \mathrm{eV}\right)$. The first attempt to grow this material at a low temperature $\left(200^{\circ} \mathrm{C}\right)$ was very promising: the resistivity of the layers was as large as $8.8 \cdot 10^{3} \Omega \cdot \mathrm{cm}$, and the electron mobility was also fairly high $\left(2260 \mathrm{~cm}^{2} / \mathrm{V} \cdot \mathrm{s}\right)$ [99]. However, carrier trapping time in this material was rather long - approximately 7 ps. Better material parameters were achieved at a lower growth temperature of $\sim 180^{\circ} \mathrm{C}$ and at reduced anneal temperatures [105]. Material with a resistivity of $\sim 10^{4} \Omega \cdot \mathrm{cm}$ and carrier lifetimes shorter than 500 fs was obtained; this was sufficient for its use in a THz-TDS system activated by $1.06 \mu \mathrm{m}$ wavelength laser pulses [106]. 


\subsubsection{Layered structures}

In several works, more complicated than the bulk layers - multiple-quantum-well (MQW) or superlattice structures for optoelectronic $\mathrm{THz}$ devices were investigated. Low-temperature growth, Be-doped, and post growth annealed InGaAs/InAlAs MQWs were shown to result in subpicosecond photoresponses and electron concentration of the order of $10^{16} \mathrm{~cm}^{-3}[107,108]$. Recently, the problem of low temperature growth of InGaAs on InP substrates was approached by combining two techniques: conventional compensation of the donors by balanced doping with Be acceptors and by embedding thin $(12 \mathrm{~nm})$ photoconductive InGaAs layers between InAlAs barriers transparent at 1.5 $\mu \mathrm{m}$ wavelength $[109,110]$. LTG InAlAs layers are known to contain a large number of deep trapping centres [99], thus the residual electrons are captured in this material, resulting in a sheet resistivity of the order of $10^{6} \Omega /$ square. Such structures were used in pulsed THz components of a TDS system with an useable frequency range $>2.5 \mathrm{THz}$ [109] and in CW optical mixing system operated at frequencies up to $1.6 \mathrm{THz}$ [110]; both systems were using $1.5 \mu \mathrm{m}$ wavelength laser sources.

An alternative structure based on InGaAs/InGaNAs supperlattice grown on InP substrate was investigated in [111]. Diluted amounts of N (up to 14\%) were introduced into InGaAs; they were narrowing theenergy bandgap and were creating numerous electron traps. The electrons excited in the InGaAs layer were trapped after their diffusion into the neighbouring InGaNAs layers. However the electron trapping times in such structures were larger than 6 ps even for the thinnest absorption layers of $2.5 \mathrm{~nm}$.

Ultrafast photoresponse was recently obtained also by embedding semi-metallic ErAs nanoparticles into InGaAs matrix to form a superlattice [112]. This unique technology was pioneered in the late 1990s by the group of A.C. Gossard and involved incorporation of Er during the normal growth of GaAs [113]. Under certain conditions, erbium was shown to form crystalline nanoparticles embedded in a nearly defect-free GaAs, and this material demonstrated subpicosecond carrier lifetimes [114]. In the first attempt to grow ErAs: $\operatorname{In}_{0.53} \mathrm{Ga}_{0.47} \mathrm{As}$ [114], the carrier trapping layers were separated by $40 \mathrm{~nm}$ and shown the lifetime of the order of $1 \mathrm{ps}$. Even though the material was Be doped, its resistivity was still low. Fig. 17 shows the vertical crosssection of InGaAs/ErAs supperlattice that has demonstrated the best device-related parameters: the dark resistivity of $3.43 \cdot 10^{2} \Omega \cdot \mathrm{cm}$ and the carrier lifetime of $220 \mathrm{fs}$ [112]. It contained a periodic sequence of self-assembled ErAs nanoparticle layers, $\mathrm{In}_{0.53} \mathrm{Ga}_{0.47}$ As spacer layers $(5 \mathrm{~nm}$ thick, for the case of the shortest lifetime), and Be $\delta$-doping in the immediate vicinity of the nanoparticle layer to compensate for the free electrons. 


\subsubsection{GaBiAs}

The search for the materials photosensitive in the wavelength range from 1 to $1.5 \mu \mathrm{m}$ that could be used in photoconducting $\mathrm{THz}$ components was not limited to InGaAs and the structures of the related semiconductor compounds. $\mathrm{GaAs}_{0.6} \mathrm{Sb}_{0.4}$ grown on GaAs substrates at temperature of $170^{\circ} \mathrm{C}$ had high resistivity after annealing and was used for manufacturing photoconductive $\mathrm{THz}$ emitters in [115]. Though the bandgap of this material was only $0.86 \mathrm{eV}$, these emitters, when activated by Ti:sapphire laser pulses, have shown much worse performance as LTG GaAs devices.

$\mathrm{GaBiAs}$ alloy was, for the first time, mentioned as a possible material for optoelectronic $\mathrm{THz}$ applications in 2006 [116]. Before that, there had been several attempts to grow GaBiAs on GaAs substrates by MBE and MOCVD techniques. The main purpose of these efforts was to produce a material with weaker, compared to other III-V compounds, temperature dependence of bandgap energy for the applications in diode lasers [117, 118]. It has been found [119] that the bandgap of GaBiAs decreases with the addition of $\mathrm{Bi}$ up to 7 times faster as compared with InAs case in InGaAs. The lattice mismatch between GaBiAs alloy and GaAs substrate is approximately four times smaller compared to InGaAs with a similar bandgap.

Because $\mathrm{Bi}$ is a large atom as compared to both $\mathrm{Ga}$ and $\mathrm{As}$, it has a tendency of a surface segregation and is difficult to incorporate into the crystalline lattice under standard MBE growth conditions. Therefore, it is necessary to grow GaBiAs at temperatures much lower than $\sim 600^{\circ} \mathrm{C}$ typical for MBE growth of GaAs [120]. Reduced growth temperature could, as it is in the case of LTG GaAs, lead to the presence of structural defects acting as fast traps for non-equilibrium current carriers, which would impair the performance of light emitters made from GaBiAs layers but could be advantageous for ultrafast photoconductive devices manufactured on their basis [116].

Figure 18 shows the energy bandgap of $\mathrm{GaBi}_{\mathrm{x}} \mathrm{As}_{1-\mathrm{x}}$ as a function of the $\mathrm{BiAs}$ content in the layer [119]. As can be seen the energy bandgap decreases with the increase of Bi content at the rate approximately equal to $-62 \mathrm{meV} / \% \mathrm{Bi}$. A similar shift has been reported earlier [121]. The larger corresponding parameter for $\operatorname{In}_{\mathrm{x}} \mathrm{Ga}_{1-\mathrm{x}} \mathrm{As}$ alloy is $-12 \mathrm{meV} / \% \mathrm{In}$ [122].

It has been shown in [123] that large bandgap shift in $\mathrm{GaBi}_{\mathrm{x}} \mathrm{As}_{1-\mathrm{x}}$ can be explained by a valence band anti-crossing caused by the interaction between the extended states in GaAs valence band and resonant $\mathrm{T}_{2}$ states of the $\mathrm{Bi}$ atoms. Comparing with GaNAs alloy, whereas similar bandgap shift is caused by a conduction band anti-crossing [124], the low-temperature-grown GaBiAs is more suitable for photoconductive applications. Nitrogen creates resonant donor levels in GaNAs and causes reduction of the dark resistivity of the layers, whereas the acceptor levels 
introduced by $\mathrm{Bi}$ in $\mathrm{GaBiAs}$ can enhance the compensation of $\mathrm{As}_{\mathrm{Ga}}$ donors created during the growth of this alloy at low temperatures and can increase both the dark resistivity and the electron trapping rate. Moreover, the lowest conduction band sub-band in GaNAs is characterized by very low electron mobility [125], in contrast with GaBiAs, where the conduction band states do not mix with the impurity states and the effect of the latter states on the electron mobility is minimal. The electron mobility in $\mathrm{GaBi}_{\mathrm{x}} \mathrm{As}_{1-\mathrm{x}}$ layers was determined from the optical pump - $\mathrm{THz}$ probe experiments [126, 127]. It has been found to be in the range from $2000 \mathrm{~cm}^{2} / \mathrm{Vs}$ to $2800 \mathrm{~cm}^{2} / \mathrm{Vs}$ much larger than the electron mobility in LTG GaAs and in diluted GaNAs alloys. Slightly lower electron mobilities were obtained from the Hall-effect measurement on heavily n-doped GaBiAs [128].

Photoexcited carrier relaxation in GaBiAs layers has been studied in the author's laboratory by the optical pump - THz probe technique based on $\mathrm{Yb}: \mathrm{KGW}$ laser pulses (the wavelength of $1030 \mathrm{~nm}$, the pulse duration of $70 \mathrm{fs}$, and the pulse repetition rate of $76 \mathrm{MHz}$ ). Fig. 19 presents the results for $\mathrm{GaBi}_{0.04} \mathrm{As}_{0.96}$ layer. The symmetrical shape of the optically induced $\mathrm{THz}$ absorption transient evidences that the electron trapping time in this material is shorter than temporal resolution of the experiment ( $\sim 800 \mathrm{fs})$. This layer was used for manufacturing photoconductive antennae that were used for $\mathrm{THz}$ pulse generation and detection in a TDS system activated by femtosecond $\mathrm{Yb}: \mathrm{KGW}$ laser pulses. Signal-to-noise ratio of $\sim 60 \mathrm{~dB}$ and frequency bandwidth more than $4 \mathrm{THz}$ are typical values for the system.

\section{Semiconductor surface emitters}

\subsection{Mechanisms of the surface THz emission}

The last Section of this review we will describe the effect of $\mathrm{THz}$ pulse emission from semiconductor surfaces illuminated by a femtosecond laser. As it was already mentioned in the Introduction, this effect is present in the majority of semiconductors, with a degree depending both on the material parameters and on the laser photon energy. In the first experiments, when $620 \mathrm{~nm}$ wavelength pulses from a femtosecond colliding-pulse mode-locked dye laser were used, the $\mathrm{THz}$ pulses were radiated by a semi-insulating InP crystal most efficiently [21]. Amplitudes of the THz pulses generated from the surfaces of various semiconductors after their illumination by more

popular presently femtosecond lasers: Ti:sapphire $(\lambda \approx 800 \mathrm{~nm})$ and $\mathrm{Yb}: \mathrm{KGW}(\lambda \approx 1030 \mathrm{~nm})$ are compared on Fig. 20. At these wavelengths (as well as at $1550 \mathrm{~nm}$ ), the $\mathrm{THz}$ pulses are most efficiently generated at the surface of a p-type InAs crystal. 
Generally speaking, the $\mathrm{THz}$ radiation at the photoexcited semiconductor surface can be emitted by a dipole that is induced either due to a fast changing photocurrent and by the nonlinear optical polarization of the material. In the far field region, the $\mathrm{THz}$ field can be expressed as

$$
E_{T H z}=-\frac{S}{c^{2} R} \int_{0}^{\infty}\left(\frac{\partial j}{\partial t}+\frac{\partial^{2} P}{\partial t^{2}}\right) d z
$$

where $c$ - is the speed of light in vacuum, $R$ - is the distance from the point of observation to the emitting region, $S$ - is the area of the laser excited spot on the semiconductor surface; $j$ and $P$ - are the photocurrent and nonlinear polarization components in the direction of the $\mathrm{THz}$ wave polarization. The integration in the relation (7) is carried out over the depth $z$. It is important to point out that the expression (7) is correct when the diameter of the illuminated spot is smaller then the wavelength of the emitted $\mathrm{THz}$ radiation, otherwise the dipole approach is not correct and the $\mathrm{THz}$ field amplitude should be determined through summation of the partial waves emitted by the separate surface elements with their phase relations taken into account. The following discussion considers different physical mechanisms of the transient photocurrent and low-frequency nonlinear polarization components in terms of the relation (7).

The electrons and holes that are optically generated in the semiconductor due to the absorption of femtosecond laser radiation are spatially separated by the built-in electric field and cause a transient photocurrent on a subpicosecond time scale. This fast varying photocurrent can be an effective source of $\mathrm{THz}$ radiation with the amplitude defined by the time derivative of the photocurrent [21]. The mechanism of $\mathrm{THz}$ generation, known as photocurrent surge effect, is observed in semiconductors with a strong surface band bending.

In the so-called photo-Dember effect, the spatial separation of the electrons and the holes occurs even when the surface field is absent [129]. The photoexcited electrons have a higher mobility and surpass less mobile holes when they diffuse from their excitation point at the surface towards the bulk of the material. As a result, the space charge and the electric field appear. Eventually, this electric field starts to slow down the electrons and to accelerate the holes, therefore, both types of carriers are finally moving almost together as a single quasi-neutral packet. The classical Dember effect [130] is usually being studied under a stationary photoexcitation when the drift-diffusion approach is correct. In this case, the Dember voltage $V_{D}$ is described by the relation:

$$
V_{D}=\frac{k_{B} T}{e} \cdot \frac{b-1}{b+1} \cdot \ln \left[1+\frac{(b+1) \Delta n}{n_{0} b+p_{0}}\right]
$$


( $b=\mu_{n} / \mu_{p}$ is the electron and hole mobility ratio, $n_{0}, p_{0}$, and $T$ are the initial electron and hole densities and the electron temperature, respectively). When the electron temperature is the same as that of the lattice, $V_{D}$ typically is low, of the order of $10^{-2} \mathrm{~V}$; it becomes approximately one order of magnitude larger, if one assumes that $T$ is equal to the hot, photoexcited electron temperature ( $T=2 \varepsilon_{e x} / 3 k_{B}$, where $\varepsilon_{e x}$ is the excess energy of the photoexcited electrons). However, in the majority III-V and IV-VI narrow-gap materials the characteristic time of the electron-LO phonon scattering is around $200 \mathrm{fs}$, these scattering processes lead only to small changes in electron momentum, therefore, during the first few hundreds of femtoseconds after the excitation, the electron movement is purely ballistic rather than diffusive. As a result, the surface photovoltages in those materials can be quite large, comparable with $\varepsilon_{e x} / e$, which can be of the order of $1 \mathrm{~V}$ and more [131].

The lowest order nonlinear optical response of a non-centrosymmetric crystal is defined by its second-order susceptibility $\chi_{2}$ and leads to the sum and difference frequency generation. In the case when the optical beam contains nearly the same frequencies (which is typical for femtosecond laser spectra) and interacts nonlinearly with the crystal, the difference frequency is in the $\mathrm{THz}$ range and the induced polarization is referred to as the optical rectification (OR). The charge displacement induced by the OR effect follows the optical pulse envelope. When a built-in dc field $E_{s}$ is present at the semiconductor surface, the transient $\mathrm{THz}$ polarization can also be induced due to the third order nonlinear susceptibility $\chi_{3}$; the $\mathrm{THz}$ pulse magnitude generated due to this electrical-fieldinduced optical rectification (EFIOR) effect will be proportional to the effective second order susceptibility $\chi_{2}^{*} \propto \chi_{3} E_{s}$. The coherent THz pulse emission due to OR effect was rather early documented both in bulk semiconductors [132] and in quantum well structures [133].

The dependence of the emitted $\mathrm{THz}$ pulse amplitude on the orientation of the optical field with regard to the crystallographic axes is usually considered as a fingerprint of nonlinear optical $\mathrm{THz}$ pulse generation mechanism. These so-called azimuthal angle $\phi$ dependences of the $\mathrm{THz}$ emission efficiency from zinc-blende semiconductors like GaAs measured at different crystallographic planes can also help in distinguishing OR and EFIOR mechanisms [134, 135]. The azimuthal angle dependences of the $\mathrm{THz}$ pulse amplitude radiated due to these two effects from differently oriented surfaces of zinc-blende crystals are listed in Table I.

Valence band of III-V semiconductors consists of two sub-bands of heavy and light holes at the centre of the Brillouin zone. Due to the optical selection rules, the momenta of electrons excited 
from the heavy-hole band by linearly polarized light will mainly lie in the plane perpendicular to the direction of the light electrical field (Fig. 21). On the other hand, the momentum distribution for electrons excited from the light-hole band will be stretched out along the direction of the optical field polarization. When the light is falling into air-semiconductor interface at an incline angle and is absorbed in a thin surface layer, the electrons moving to the right in Fig. 21 will be diffusively scattered by the surface and a lateral photocurrent component will arise. This lateral photocurrent effect has been discovered in GaAs crystals in [136]. In this investigation as well as in another experiment when polarized hot-electron photoluminescence was observed [137], cryogenic temperatures and photon energies close to the energy bandgap were used for reducing the electron scattering rate. However, when the semiconductor is excited by a short light pulse, such lateral surface photocurrent component will be present even at the room temperature during the first few hundreds of femtoseconds when the electrons are still moving ballisticaly. Fast changing lateral photocurrent transient could lead to the $\mathrm{THz}$ radiation that will be more efficiently out-coupled from the semiconductor than the radiation originating from the carrier movement in the direction perpendicular to the surface. Indirect proof of this optical orientation effect has been found recently by measuring the angular dependences of the THz radiation from InAs [138]. It was observed there that the radiating dipole is tilted from the normal to the surface and coincides with the direction of the optical beam refracted inside the crystal.

Coherent plasmon or coherent phonon generation can also influence the characteristics of $\mathrm{THz}$ emission from semiconductor. Fast changes of surface electric field can initiate plasma oscillations of the intrinsic carriers that will amplify the THz wave [139]. On the other hand, pulsed excitation of the semiconductor can result in coherent generation of infrared-active lattice vibrations that will produce oscillations of macroscopic dielectric polarization and emission of electromagnetic waves at the phonon frequency. Such an effect has been observed in Te [140] and several other semiconductors.

When considering $\mathrm{THz}$ emission from the surfaces of particular semiconductor materials, simultaneous action of several physical mechanisms should be taken into account. In the following we will discuss the main features of this effect in several materials in more detail.

\subsection{InAs and other narrow-gap semiconductors.}

Generally speaking, semiconductors with a narrow energy bandgap should be better $\mathrm{THz}$ emitters than the large bandgap materials. The narrow-gap semiconductors are usually characterized by high electron mobility, thus, when excited by femtosecond laser pulses, they exhibit stronger 
surface current surge effects. Moreover, the optical nonlinearities in these materials are, as a rule, also larger than in wide-gap semiconductors.

The first narrow-gap semiconductor, which has attracted researchers' attention as a promising $\mathrm{THz}$ surface emitter, was InSb. It has been found in [141] that it radiates $\mathrm{THz}$ pulses when photoexcited with $1.9 \mu \mathrm{m}$ wavelength femtosecond laser. At low temperatures the emitted $\mathrm{THz}$ pulse energy at this excitation wavelength was nearly two orders of magnitude larger than when this wavelength was equal to $800 \mathrm{~nm}$. Later on, the same group has obtained well defined azimuthal angle dependences on (111) crystallographic planes [142], which has evidenced a significant contribution of the nonlinear optical mechanisms of surface $\mathrm{THz}$ emission from InSb.

Strong impulse for investigations of narrow-gap semiconductors was provided by the discovery of unusual $\mathrm{THz}$ emission properties of InAs. When photoexcited with Ti:sapphire laser pulses $(800 \mathrm{~nm})$ [143], InAs radiates 10 times larger amplitude $\mathrm{THz}$ pulses than GaAs - the best known $\mathrm{THz}$ emitter at that time. Moreover, the radiated THz power increased by almost 100 times when $1 \mathrm{~T}$ magnetic field was applied in parallel to the InAs sample's surface. A large number of investigations focused on the mechanism of $\mathrm{THz}$ emission from InAs. It was found that this material efficiently radiated $\mathrm{THz}$ pulses also when photo-excited with $1.550 \mu \mathrm{m}$ laser [144]. McLaughlin et al. [145] reported a quadratic dependence of the $\mathrm{THz}$ emission on magnetic field up to $3 \mathrm{~T}$. Beyond $3 \mathrm{~T}$, Ohtake et al. [146] demonstrated that the radiated $\mathrm{THz}$ power saturates at $4 \mathrm{~T}$, decreases to a minimum at $6 \mathrm{~T}$, and increases again at $14 \mathrm{~T}$.

Initially, the photo-Dember effect caused by different photoexcited electron and hole diffusion rates was considered as the main mechanism responsible for the $\mathrm{THz}$ emission from InAs surfaces [147]. The experimentally observed enhancement of $\mathrm{THz}$ power with magnetic field was explained by a reorientation of the THz dipole with respect to the surface [148]. No azimuthal angle dependences were recorded on (100) crystallographic planes, for which the majority of the experiments were performed; considerable azimuthal angle dependent effect observed on (111) plane was assumed to be of importance only for this crystallographic orientation [147]. Possible influence of the surface electric field was usually also ignored due to a narrow bandgap of InAs and potentially small band-bending at its surface. This is not always justifiable, because surface potential in InAs is fixed at fairly high $(\sim 0.2 \mathrm{eV}$ [149]) energies above the conduction bend edge while the surface inversion layer can be sufficiently wide and strong in a p-doped crystal.

New experimental facts emerged after discovery [150] that a p-type InAs is a better $\mathrm{THz}$ emitter than an n-type InAs. This fact was explained in [150] as a consequence of the EFIOR effect induced by the surface field in the p-InAs. Later on, analysis of the symmetry of the azimuthal angle dependences of $\mathrm{THz}$ radiation, which was performed in [134] by illuminating different 
crystalline planes of n-InAs, pointed out to the predominance of the EFIOR effect in n-doped crystals as well, although the origin of the surface electric field remained unclear in this case. A mechanism of $\mathrm{THz}$ generation in InAs that took into account both photo-Dember and EFIOR contributions was proposed in [151]. It was suggested there that the surface field was induced due to the photo-excited electron and hole separation and caused the effective second-order optical nonlinearity responsible for the EFIOR effect. This suggestion has been supported experimentally by double-pulse excitation of the sample and through Monte-Carlo simulation [151].

Ternary $\mathrm{Cd}_{1-\mathrm{x}} \mathrm{Hg}_{\mathrm{x}} \mathrm{Te}$ alloys and $\mathrm{InSb}$ have smaller bandgaps and electron effective masses than InAs, therefore one would expect that these materials should be even more efficient $\mathrm{THz}$ emitters. However, experiments have shown than average $\mathrm{THz}$ power radiated from the surfaces of InSb and $\mathrm{Cd}_{1-\mathrm{x}} \mathrm{Hg}_{\mathrm{x}} \mathrm{Te}$ with $\mathrm{x}=0,0.2$, and 0.3 is more than two orders of magnitude smaller than from the surface of InAs $[147,152]$. The reduced efficiency of $\mathrm{THz}$ emission from InSb was explained by photoexcited electron transfer to subsidiary conduction band $\mathrm{L}$ valleys with a large electron effective mass [147], whereas in the case of $\mathrm{Cd}_{1-\mathrm{x}} \mathrm{Hg}_{\mathrm{x}} \mathrm{Te}$ the main reason of weak $\mathrm{THz}$ emission is, most probably, large non-parabolicity of the conduction band [153].

The electron energy dispersion relation in narrow-gap semiconductors is often described by the Kane's law [154]:

$$
\frac{\hbar^{2} k^{2}}{2 m_{e 0}}=\varepsilon(1+\alpha \varepsilon),
$$

where $m_{e 0}$ is the effective mass at the bottom of the conduction band $\mathcal{E} \approx 1 / \varepsilon_{g}$ is the nonparabolicity coefficient. According to this law, the electron effective mass increases with its energy in the conduction band as:

$$
m_{e}(\varepsilon)=m_{e 0}(1+2 \alpha \varepsilon) .
$$

For example, in $\mathrm{Cd}_{0.2} \mathrm{Hg}_{0.8} \mathrm{Te}$ with $\varepsilon_{g}=0.1 \mathrm{eV}$ the electrons excited with a Ti:sapphire laser start with the effective mass 28 times larger that its value at the thermal equilibrium, therefore their movement will be significantly impaired. Moreover, at room temperature, both $\mathrm{InSb}$ and $\mathrm{Cd}_{1-}$ ${ }_{x} \mathrm{Hg}_{\mathrm{x}} \mathrm{Te}$ contain high intrinsic carrier densities responsible for an increased screening of the surface photovoltage caused by illumination with the femtosecond laser pulses. Consequently, the $\mathrm{THz}$ emission is less efficient because of screening. At lower temperatures, $\mathrm{THz}$ emission from these semiconductors shows a significant growth (Fig. 22) and becomes comparable with that of InAs [155]. 
Surface $\mathrm{THz}$ emission has turned up to be unique tool for measuring important semiconductor parameter - the energy separation $\Delta \varepsilon$ between the main $\Gamma$ and the subsidiary $L$ or $X$ valleys in the conduction band. The velocity, at which photoexcited electrons move quasi-ballistically from the surface towards the bulk of the crystal, the resultant electron and hole separation, and the radiated $\mathrm{THz}$ signal will increase with the photon energy until the excess energy reaches the position of the subsidiary conduction band valleys. The electron inter-valley scattering effectively disturbs the quasi-ballistic movement. As a result, the $\mathrm{THz}$ pulse amplitude starts decreasing at large photon energies, and the $\mathrm{THz}$ excitation spectrum has a maximum that can be used for determining the inter-valley energy separation in the conduction band. Such experiment has been, for the first time, performed in [156] with tuneable wavelength femtosecond pulses. Results of this experiment are presented in Fig. 23; their analysis has led to the estimation of the inter-valley energy separation in $\operatorname{InSb}\left(\Delta \varepsilon_{\Gamma L}=0.53 \mathrm{eV}\right)$ and $\operatorname{InAs}\left(\Delta \varepsilon_{\Gamma L}=1.06 \mathrm{eV}\right)$.

It becomes clear from the discussion presented above that the exceptional properties of InAs as a $\mathrm{THz}$ emitter are determined by several factors simultaneously. First of all, Ti:sapphire laser excites the electrons just below the energy position of the L valleys, therefore they are moving at the highest possible velocity. Secondly, the energy bandgap of this material is not narrow enough to cause large conduction band non-parabolicity and too high intrinsic carrier densities. Finally, the built-in electric field at the surface of p-type InAs crystals has the same polarity as the field induced by the photoexcited electron and hole separation, which is leading to more efficient $\mathrm{THz}$ pulse generation due to the EFIOR effect. Although InAs is probably the best surface THz emitter at 800 nm wavelength, at longer wavelengths it could be surpassed by other narrow-gap semiconductors. As it can be seen from THz excitation spectra presented on Fig. 23, the efficiency of THz emission from InSb surface is larger than that from InAs in the wavelength range around $1.5 \mu \mathrm{m}$.

\subsection{Other semiconductors}

When analyzing physical mechanisms responsible for $\mathrm{THz}$ emission from different materials it is interesting to note that several phenomena can take place in a single semiconductor simultaneously. For GaAs excited with laser quanta smaller that its band gap $\varepsilon_{g}$, the $\mathrm{THz}$ emission is caused by the optical rectification effect [157]. When the photon energy is comparable to $\varepsilon_{g}$, the main physical mechanism is the photocurrent surge in the surface electric field [21]; at even larger photon energies, the EFIOR effect starts to dominate the THz pulse emission [158]. Additionally, in n-doped GaAs cold electron plasma oscillations were found to contribute to the $\mathrm{THz}$ emission [159]. Relatively strong THz emission can be observed from all alloys of the GaAs-InAs family; its 
amplitude is increasing with increasing InAs part in the alloy $[160,161]$ and grows proportionally to the electron excess energy.

Stronger or weaker $\mathrm{THz}$ radiation was observed from the surfaces of practically all semiconductors illuminated by femtosecond Ti:sapphire laser. Exception is crystalline silicon, for which the direct gap $(2.5 \mathrm{eV})$ is larger than the photon energy of this laser. However, some $\mathrm{THz}$ emission has been documented in highly absorptive version of this material, so called "black silicon" [162]. The $\mathrm{THz}$ emission has been observed from the surfaces of other elemental semiconductors: tellurium [139] and germanium. In the latter material contributions of both the photo-Dember effect [163] and the EFIOR effect [164] are present with significant interplay of both these physical mechanisms [165]. As Ge crystal is centre-symmetrical and its second order optical susceptibility is zero, the optical rectification effect, which can be unambiguously identified from the azimuthal angle dependences of THz emission presented on Fig. 24, is caused solely by EFIOR.

Recently, after finding out that energy bandgap of $\mathrm{InN}$ is much narrower than it was assumed before, $\mathrm{THz}$ emission from this material has been studied in several investigations [166-168] by with Ti:sapphire laser pulses. Vertically aligned nanorod structures [167] and the use of the samples cut parallel to the a-plane [168] have led to some enhancement of $\mathrm{THz}$ radiation from $\mathrm{InN}$; nevertheless, even in the best cases the power of this radiation was more than 10 times weaker that from n-InAs.

\section{Conclusions}

Investigations of semiconductor materials used in optoelectronic terahertz frequency range emitters and detectors have been reviewed. It was noted that this field of applications requires materials with an exceptional set of material parameters: large resistivity, high electron mobility, and ultrashort, of the order of $1 \mathrm{ps}$ and shorter, electron lifetimes. At first, such a set of parameters was discovered in low-temperature MBE grown GaAs and other highly non-stoichiometric variants of this material; these semiconductors are presently successfully used in THz-TDS systems activated by femtosecond Ti:sapphire laser pulses. Recently, a significant progress was also achieved in investigating semiconductor materials for optoelectronic $\mathrm{THz}$ devices that could be activated by optical pulses of a longer wavelengths generated by more compact than Ti:sapphire laser systems. Devices manufactured from multi-layer structures based on InGaAs and on lowtemperature $\mathrm{GaBiAs}$ layers have already demonstrated remarkable performance.

Last decade was also marked by a growing interest in $\mathrm{THz}$ pulse emission from the unbiased semiconductor surfaces illuminated by femtosecond laser pulses, which can be an alternative to the photoconductive switches. Several different physical mechanisms can contribute to this effect, 
which makes surface emission a rather universal phenomenon in semiconductors. As yet, the most efficient $\mathrm{THz}$ emitter is p-type InAs crystal. In this material the narrow bandgap and the large intervalley separation in the conduction band are combined with the moderate non-parabolicity, sufficient band-bending at the surface, and the large nonlinear optical susceptibilities that result in simultaneous action of several physical effects responsible for the THz pulse emission.

\section{Acknowledgements}

Discussions and collaborations with numerous colleagues world-wide are highly appreciated and especially Professors J L Coutaz, C. Jagadish, M. Kaminska, and S. Marcinkevičius should be mentioned together with Drs. R. Adomavičius, K. Bertulis, and V. Pačebutas. The author wishes to thank Prof. A. Matulionis for careful reading of the manuscript and valuable suggestions. The work was in part supported by the Lithuanian Science and Study Foundation under the grant B12-2009. 


\section{References}

[1] Mittleman D ed 2003 Sensing With Terahertz Radiation (ser. Opt. Sci., Springer-Verlag, Berlin)

[2] Miles R E, Harrison P and Lippens D ed 2000 Terahertz Sources and Systems (NATO Science Series, Kluwer, Dordrecht)

[3] Miles R E, Zhang X-C, Eisele H. and Krotkus A ed 2007 Terahertz Frequency Detection and Identification of Materials and Objects (NATO Science for Peace and Security Series, Springer, Dordrecht)

[4] Dexheimer S L ed 2008 Terahertz Spectroscopy - Principles and Applications (CRC Press, Boca Raton)

[5] Davies A G, Linfield E H and Johnston M B 2002 Physics in Medicine and Biology 473679

[6] Mocker H and Collins R 1965 Appl. Phys. Lett. 7270

[7] Auston D H 1975 Appl. Phys. Lett. 26101

[8] Lee Ch H ed 1984 Picosecond Optoelectronic Devices (Acad. Press, Orlando)

[9] Mourou G and Knox W 1980 Appl. Phys. Lett. 36623

[10] Mourou G, Knox W H and Williamson S 1984 in Picosecond Optoelectronic Devices, ed. Lee Ch H (Academic Press) p. 219

[11] Krotkus A 1990 Int. J. Optoelectr. 5483

[12] Smith P R, Auston D H and Augustyniak W M 1981 Appl. Phys. Lett. 39739

[13] Valdmanis J A and Mourou G 1984 in Picosecond Optoelectronic Devices, ed. Lee Ch H (Academic Press) p. 249

[14] Fork R L, Green B J and Shank C V 1981 Appl. Phys. Lett. 38671

[15] Auston D H, Cheung K P and Smith P R 1984 Appl. Phys. Lett. 45284

[16] Fattinger Ch and Grischkowsky D 1989 Appl. Phys. Lett. 54490

[17] Van Exter M, Fattinger Ch and Grischkowsky D 1989 Appl. Phys. Lett. 55337

[18] Van Exter M, Fattinger Ch and Grischkowsky D 1989 Opt. Lett. 141128

[19] Grischkowsky D, Keiding S, van Exter M and Fattinger Ch, 1990 J. Opt. Soc. Am. B7 2006

[20] Smith F W, Lee H Q, Diadiuk V, Hollis M A, Calawa A R, Gupta S, Frankel M, Dykaar D R, Mourou G A and Hsiang T Y 1989 Appl. Phys. Lett. 54890

[21] Zhang X C, Hu B B, Darrow J T, Auston D H 1990 Appl. Phys. Lett. 561011

[22] Malevich V L, Adomavičius R and Krotkus A 2008 C. R. Physique 9130

[23] Leitensdorfer A, Hunsche S, Shah J, Nuss M C and Knox W H 2000 Phys. Rev. B61 16642

[24] Duvilaret L, Garet F, Roux J-F and Coutaz J-L 2001 IEEE J. Sel. Top. Quantum Electron 7615

[25] Uhd Jepsen P, Jacobsen R H and Keiding S R 1996 J. Opt. Soc. Am. B 132424

[26] Krokel D, Grischkowsky D and Ketchen M B 1989 Appl. Phys. Lett., 54, 1046

[27] Sun F G, Wagoner G A and Zhang X C 1995 Appl. Phys. Lett. 671656

[28] Kono S, Tani M and Sakai K 2001 Appl. Phys. Lett. 79898

[29] Kono S, Tani M and Sakai K 2002 IEE Proc.-Optoelectronics 149105

[30] Ludwig Ch and Kuhl J 1996 Appl. Phys. Lett. 691194

[31] Son J-H, Norris T B and Whitaker J F 1994 J. Opt. Soc. Am. A 112519

[32] Reklaitis A, Grigaliunaite G and Krotkus A 1999 Semicon. Sc. Technol. 14945

[33] Johnson A M, Auston D H, Smith P R, Bean J C, Harbison J P and Adams A C, 1981 Phys. Rev. B23 6816

[34] Stolk P A, Saris F W, Berntsen A J M, Van det Weg W F, Sealy L T, Barklie R C, Kroetz G and Mueller G 1994 J. Appl. Phys., 75, 7266

[35] Doany F E, Grischkowsky D and Chi C C 1987 Appl. Phys. Lett. 50460

[36] Lui K P H and Hegmann F A 2003 J. Appl. Phys. 939012

[37] Fork R L, Greene B I and Shank C V 1981 Appl. Phys. Lett. 38671

[38] Spence D E, Kean P N and Sibbett W, 1991 Opt. Lett. 1642

[39] Smith F W, Calawa A R, Chen C L, Manfra M J and Mahoney L J 1988 IEEE Electron Device. Lett. 9 
[40] Smith F W, Le H Q, Diadiuk V, Hollis M A, Calawa A R, Gupta S. Frankel M, Dykaar D R, Mourou G A. and Hsiang T Y 1989 Appl. Phys. Lett. 54890

[41] Nolte D D 1999 J. Appl. Phys., 85, 6259

[42] Krotkus A and Coutaz J-L 2005 Semicond. Sci. Technol. 20 S142

[43] Kordos P, Forster A, Marso M and Ruders F 1998 Electron. Lett. 34119

[44] Loka H S and Smith P W E 1998 IEEE Photon. Technol. Lett. 101733

[45] Keller U, Weingarten K J, Kaertner F X, Kopf D, Braun B, Jung I D, Fluck R, Hoenninger C,

Matuschek N and Aus der Au J 1996 IEEE J. Sel. Top. Quantum Electron 2435

[46] Shen Y C, Upadhya P C, Linfield E H, Beere H E and Davies A G 2003 Appl. Phys. Lett. 833117

[47] Kono S, Tani M and Sakai K 2001 Appl. Phys. Lett. 79119

[48] McIntosh K A, Brown E R, Nichols K B, MsMahon O B, DiNatale W F and Lyszczarz T M 1994

Appl. Phys. Lett. 673844

[49] Stelmacher M, Bisaro R, Galtier P, Nagle J, Khirouni K and Bourgoin J C 2001 Semicond. Sc.

Technol. 16440

[50] Liu X, Prasad A, Chen W M, Kurpiewski A, Stoschek A, Liliental-Weber Z and Weber E R 1994 Appl. Phys. Lett. 653002

[51] Kaminska M and Weber E R 1991 Defects in Semiconductors, ed. Davies G, DeLeo G G and Stavola M (Trans Tech. Pennsylvania) 1033

[52] Gebauer J., Krause-Rehburg R., Eichler S., Luysberg M., Sohn H. and Weber E. R. 1997 Appl. Phys. Lett. 71638

[53] Look D C, Walters D C, Mansreh M O, Sizelove J R, Stutz C E and Evans K R 1990 Phys. Rev. B42 3578

[54] Campbell A C, Crook G E, Rogers T J, and Streetman B G 1990 J. Vac. Sci. Technol. B8 305

[55] Staab T E M, Nieminen R M, Luysberg M, Gebauers J and Frauenheim Th 2003 Physica B, 340-342 293

[56] Warren A C, Woodall J C, Freeouf J L, Grischkowsky D, McInturff D T, Melloch M R and Otsuka N 1990 Appl. Phys. Lett. 571331

[57] Luo J K, Thomas H, Morgan D V and Westwood D 1994 Appl. Phys. Lett. 653614

[58] Luo J K, Thomas H, Morgan D V and Westwood D 1996 J. Appl. Phys. 763622

[59] Smith F W, Le H Q, Diaduk V, Holis M A, Calawa A R, Gupta S, Frankel M, Mourou G A and Hsiang T Y. 1989 Appl. Phys. Lett. 54890

[60] Roux J-F, Coutaz J-L and Krotkus A 1999 Appl. Phys. Lett., 742462

[61] Siegner U, Fluck ., Zhang G and Keller U 1996 Appl. Phys. Lett. 692566

[62] Krotkus A, Viselga R, Bertulis K, Jasutis V, Marcinkevičius S and Olin U 1995 Appl. Phys. Lett. 66 1939

[63] Prabhu S S, Ralph S E, Melloch M R and Harmon E S 1997 Appl. Phys. Lett. 702419

[64] Kash J A, Tsang J C, Hwam J M, 1985 Phys. Rev. Lett. 542151

[65] Hu B B, de Souza E A,. Knox W H, Cunningham J E, Nuss M C, Kuznetsov A V and Chuang S L

1995 Phys. Rev. Lett. 741689

[66] Yano R, Hirayama Y, Miyashita S, Uesugi N and Uehara S 2003 J. Appl. Phys. 943966

[67] McIntosh K A, Nichols K B, Verghese S and Brown E R 1997 Appl. Phys. Lett. 70354

[68] Gregory I S, Baker C, Tribe W R, Evans M J, Beere H E, Linfield E H, Davies A G and Missous M

2003 Appl. Phys. Lett. 834199

[69] Krotkus A, Bertulis K, Dapkus L, Olin U and Marcinkevičius S 1999 Appl. Phys. Lett. 753336

[70] Marcinkevičius S, Krotkus A, Viselga R, Olin U and Jagadish C 1997 Semicond. Sci. Technol. 12396

[71] Loukakos P A, Kalpouzos C, Perakis I E, Hatzopolous Z, Logaki M and Fotokis C 2001 Appl. Phys. Lett. 792883

[72] Nemec H, Pashkin A, Kuzel P, Khasan M, Schnuell S and Wilke I 2001 J. Appl. Phys. 901303

[73] Zhang M H, Guo L W, Huang H W Li, Q, Bao C L, Zhou J M, Liu B L, Zhang Z Y Xu, Y H and Lu L W 2001 Phys. Rev. B63 115324

[74] Haiml M, Siegner U, Morier-Genoud F, Keller U, Lyusberg M, Specht P and Weber E R 1999 Appl.

Phys. Lett. 741269 
[75] Specht P, Lutz R C, Zhao R, Weber E R, Liu W K, Bacher K, Towner F J, Stewart T R and Lyusberg M 1999 J. Vac. Sc. Technol. B17 1200

[76] Krotkus A, Bertulis K, Kaminska M, Korona K, Wolos A, Siegert J, Marcinkevičius S, Roux J-F and Coutaz J-L 2002 IEE Proc. Optoelectron. 149111

[77] Ortiz V, Nagle J and Alexandrou A 2002 Appl. Phys. Lett. 802505

[78] Grenier P and Whitaker J F 1997 Appl. Phys. Lett. 701998

[79] Adomavičius R, Krotkus A, Bertulis K, Sirutkaitis V, Butkus R and Piskarskas A 2003 Appl. Phys.

Lett. 835304

[80] Blakemore J S 1982 J. Appl. Phys. 53 R123

[81] Krotkus A, Marcinkevicius S, Jasinski J, Kaminska M, Tan H H and Jagadish C 1995 Appl. Phys. Lett. 663304

[82] Jagadish C, Tan H H, Krotkus A, Marcinkevicius S, Korona K P and Kaminska M 1996 Appl. Phys. Lett. 682225

[83] Lederer M, Luther-Davies B, Tan H H and Jagadish C 1998 IEEE J. Quantum Electro. 342150

[84] Liu T A, Tani M and Pan C L 2003 J. Appl. Phys. 932996

[85] Lloyd-Hughes J, Castro-Camus E, Fraser M D, Jagadish C and Johnston M B 2004 Phys. Rev. B70 235330

[86] Claverie A, Namavar F and Liliental-Weber Z 1993 Appl. Phys. Lett. 621271

[87] Marcinkevičius S, Krotkus A, Jasutis V, Bertulis K, Tan H H, Jagadish C and Kaminska M 1996 Appl. Phys. Lett. 68397

[88] Jagadish C, Tan H H, Jasinski J, Kaminska M, Palczewska M, Krotkus A and Marcinkevičius S 1995 Appl. Phys. Lett. 671724

[89] Walukiewicz W, Liliental-Weber Z, Jasinski J, Almonte M, Prasad A, Haller E E, Weber E R, Grenier P and Whitaker J F 1996 Appl. Phys. Lett. 692569

[90] Miculics M, Marso M, Kordos P, Stancek S, Kovac P, Zheng X, Wu S and Sobolewski R 2003 Appl.

Phys. Lett. 831719

[91] Mangeney J, Lopez J, Stelmakh N, Lourtioz J-M, Oudar J-L and Bernas H 2000 Appl. Phys. Lett. 7640

[92] Opyd W G, Gibbons J F, Bravman J C, Parker M. A. 1986 Appl. Phys. Lett. 49974

[93] Zhang Y, Ding E and Zhang T 1999 Nucl. Instrum. Methods, B, 152, 307

[94] Geižutis A, Adomavičius R, Urbanowicz A, Bertulis K, Krotkus A, Tan H H and Jagadish C 2005

Lithuan. J. Phys 45, 249

[95] Brown E R, Macintosh K A, Nichols K B and Dennis C L 1995 Appl. Phys. Lett. 66285

[96] Kordos P, Marso M and Miculics M 2007 Appl. Phys. A87 563

[97] Tani M, Lee K-S and Zhang X. -C 2000 Appl. Phys. Lett. 771396

[98] Gupta S, Whitaker J F and Mourou G A 1992 IEEE J. Quant. Electron. 242464

[99] Metzger R A, Brown A S, McCray L G and Henige J A 1993 J. Vac. Sci. Technol. B11 798

[100] Chen Y, Prabhu S S, Ralph S E and McInturff D T 1998 Appl. Phys. Lett. 72439

[101] Kim J M, Lee Y T, Song J D, Kim J H, 2004 J. Cryst. Growth, 265, 8

[102] Mangeney J, Joulaud L, Crozat P, Lourtioz J-M and Decobert J 2003 Appl. Phys. Lett. 835551

[103] Chimot N, Mangeney J, Crozat P, Lourtioz J M, Blary K, Lampin J F, Mouret G, Bigourd D and Fertein E 2006 Opt. Express 141846

[104] Carmody C, Tan H H, Jagadish C, Gaarder A and Marcinkevičius S 2003 Appl. Phys. Lett. 82856

[105] Baker C, Gregory I S, Tribe W R, Bradley I V, Evans M J, Linfield E H and Missous M 2004 Appl. Phys. Lett. 854965

[106] Baker C, Gregory I S, Tribe W R, Bradley I V, Evans M J, Withers M, Taday P F, Wallance V P, Linfield E H, Davies A G and Missous M 2003 Appl. Phys. Lett. 834113

[107] Takahashi R, Kawamura Y and Iwamura H 1996 Appl. Phys. Lett. 68153

[108] Juodawlkis P W, McInturff D T and Ralph S E 1996 Appl, Phys. Lett. 694062

[109] Sartorius B, Roehle H, Künzel H, Böttcher J, Schlak M, Stanze D, Venghaus H and Schell M 2008

Opt. Express 169565

[110] Sartorius B, Schlak M, Stanze D, Roehle H, Künzel H, Schmidt D, Bach H-G, Kunkel R, and Schell M 2009 Opt. Express 1715001 
[111] Martin M, Mangeney J, Travers L, Minot C, Harmand J C, Mauguin O and Patriarche G, 2009 Appl. Phys. Lett., 95141910

[112] Ospald F, Maryenko D, von Klitzing K, Driscoll D C, Hanson M P, Gossard A C, Lu H L and Smet J H 2008 Appl. Phys. Lett. 92131117

[113] Kadow C, Fleischer S B, Ibbetson J P, Bowers J E and Gossard A C 1999. Appl. Phys. Lett. 753548

[114] Driscoll D C, Hanson M, Kadow C and Gossard A C 2001 Appl. Phys. Lett. 781703

[115] Sigmund J, Sydlo C, Hartnagel H L, Benker N, Fuess H, Rutz F, Kleine-Ostmann T and Koch M 2005 Appl. Phys. Lett. 87252103

[116] Bertulis K, Krotkus A, Aleksejenko G, Pačebutas V, Adomavičius R, Molis G and Marcinkevičius S 2006 Appl.Phys. Lett. 8820113

[117] Yoshimoto M, Murata S, Chayahara A, Horima Y, Saraie J and Oe K 2003 Jap. J. Appl.Phys., Part 2, 42 L1235

[118] Tixier S, Adamcyk M, Tiedje T, Francoeur S, Mascarenhas A, Wei P and Schietekatte P 2003 Appl. Phys.Lett. 822245

[119] Francoeur S, Seong M J, Mascarenhas A, Tixier S, Adamcyk M and Tiedje T 2003 Appl.Phys.Lett. 82 3874

[120] Pačebutas V, Bertulis K, Dapkus L, Aleksejenko G, Krotkus A, Yu K M and Walukiewicz W 2007

Semicond. Sc. Technol. 22818

[121] Yoshimoto M, Huang W, Feng G and Oe K 2006 Phys. Status Solidi b 71421

[122] Vurgaftman I, Meyer J R and Ram-Mohan I R 2001 J. Appl.Phys, 895815

[123] Alberi K, Dubon O D, Walukiewicz W, Yu K M, Bertulis K and Krotkus A 2007 Appl. Phys. Lett. 91 051909

[124] Zhang Y, Mascarenhas A and Wang L W 2006 Phys. Rev. B 71155201

[125] Shan W, Yu K M, Walukiewicz W, Wu J, Ager J W and Haller E E 2004 J. Phys. Cond. Mat. 16

S3355

[126] Pačebutas V, Bertulis K, Aleksejenko G and Krotkus A 2009 J. Mater. Sci: Mater. Electron. 20 S363

[127] Cook D G, Hegmann F A, Young E C and Tiedje T 2006 Appl. Phys. Lett. 89122103

[128] Kini R N, Bhusal L, Ptak A J, France R and Mascarenhas A 2009 J. Appl. Phys. 106043705

[129] Dekorsy T, Pfeifer T, Kütt W and Kurz H 1993 Phys. Rev. B 473842

[130] Dember H, 1931 Phys. Z, 32554

[131] Malevich V L, Adomavičius R and Krotkus A 2008 C. R. Physique 9130

[132] Chuang S L, Schmitt-Rink S, Griene B I, Saeta P N and Levi A F J 1992 Phys. Rev. Lett. 68102

[133] Planken P C M, Nuss M C, Knox W H, Miller D A B and Goosen K W 1992 Appl. Phys. Lett. 61

2009

[134] Reid M, Cravetchi I V and Fedosejevs R 2005 Phys. Rev. B 72035201

[135] Malevich V L, Krotkus A, Bičiūnas A and Pačebutas V 2008 J. Appl. Phys. 104113117

[136] Alperovich V L, Belinicher V I, Novikov V N and Terechov A S 1980 JETP Pisma 31581

[137] Zemskii V I, Zacharchenia B P and Nirlin D N 1976 JETP Pisma 2496

[138] Inoue R, Takayama K and Tonoushi M 2009 J. Opt. Soc. Am. B 26 A14

[139] Kersting R, Ulterrainer K, Strasser G, Kaufmann H F and Gornik E 1997 Phys. Rev. Lett. 793038

[140] Dekorsy T, Auer H, Waschke C, Bakker H J, Roskos H G, Kurz H, Wagner V and Grosse P 1995

Phys. Rev. Lett. 74, 738

[141] Howells B C, Herrera S D and Schlie L A 1994 Appl. Phys. Lett. 652946

[142] Howells B C and Schlie L A 1995 Appl. Phys. Lett. 673688

[143] Sarukura N, Ohtake H, Izumida S and Liu Z 1998 Appl. Phys. Lett. 84, 654

[144] Kondo T, Sakamoto M, Tonoushi M and Hangyo M 1999 Jpn. J. Appl. Phys. 38 L1035

[145] McLaughlin R, Corchia A, Johnston M B, Chen Q, Ciesla C M, Arnone DD, Jones G A C, Linfield E

H, Davies A G, Pepper M 2000 Appl. Phys. Lett. 762038

[146] Ohtake H, Murakami H, Yano T, Ono S, Sarukura N, Takahashi H, Suzuki Y, Nishijima G, Watanabe K 2003 Appl. Phys. Lett. 821164

[147] Gu P, Tani M, Kono S, Sakai K and Zhang X-C 2002 J. Appl. Phys. 915533 
[148] Johnston M B, Whittaker D M, Corchia A, davies A G and Linfield E H 2002 Phys. Rev. B65 165301 [149] Affentaugschegel C and Wieder H H 2001 Semicond. Sci. Technol. 16708

[150] Adomavičius R, Urbanowicz A, Molis G, Šatkovskis E and Krotkus A 2004 Appl. Phys. Lett. 852463

[151] Krotkus A, Adomavičius R, Molis G and Malevich V L 2007 J. Nanolectron. Optoelectron. 2108

[152] Krotkus A, Adomavičius R, Molis G, Urbanowicz A and Eusebe H 2004 J. Appl. Phys. 96, 4006

[153] Adomavičius R, Šustavičiūte R and Krotkus A 2007 Proceed. 13th International Conf. Narrow-Gap Semicond. Guilford, England, 41

[154] Kane E.1957 J. Phys. Chem. Solids 1249

[155] Molis G, Adomavičius R and Krotkus A 2008 Physica B 4033786

[156] Adomavičius R, Molis G, Krotkus A and Sirutkaitis V 2005 Appl.Phys. Lett. 87035201

[157] Zhang X -C, Jin Y, Yang K, Schowalter L J 1992 Phys. Rev. Lett. 692303

[158] Molis G, Krotkus A and Vaičaitis V 2009 Appl. Phys. Lett. 94091104

[159] Kersting R,. Unterrainer K, Strasser G, Kauffmann H F and Gornik E. 1997 Phys. Rev. Lett. 793038

[160] Malevich V L, Adomavičius R and Krotkus A 2008 C. R. Physique 9130

[161] Ko Y, Sengupta S, Tomasulo S, Dutta P and Wilke I 2008 Phys. Rev. B78 035201

[162] Hoyer P, Theuer M, Beigang R and Kley E-B 2008 Appl. Phys. Lett. 93091106

[163] Urbanowicz A, Adomavičius R, Krotkus A and Malevich V L 2005 Semicond. Sci. Technol. 201010

[164] Urbanowicz A, Krotkus A, Adomavičius R and Malevich V L 2007 Physica B: Condens. Matter 398 98

[165] Bičiūnas A, Pačebutas V and Krotkus A 2009 Physica B: Condens. Matter 4043386

[166] Ascazubi R, Wilke I, Denniston K, Lu H L and Schaff W J 2004 Appl. Phys. Lett. 844810

[167] Ahn H, Shen C-H, Wu C-L, and Gwo S. 2005 Appl. Phys. Lett. 86201905

[168] Ahn H, Ku Y-P, Chuang C-H, Pan C-L, Lin H-W, Hong Y-L and Gwo S 2008 Appl. Phys. Lett. 92 102103 


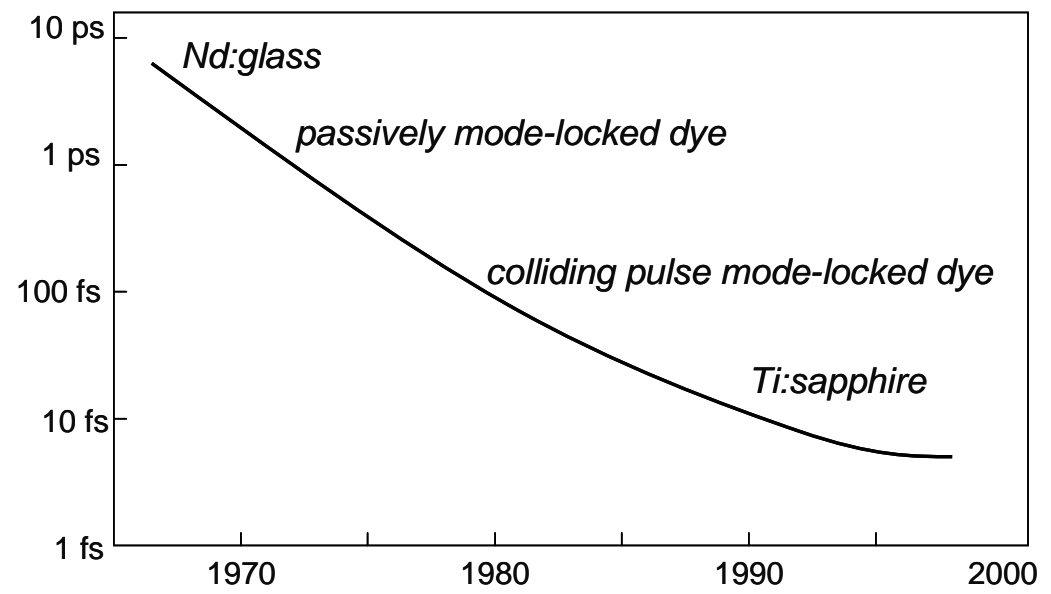

Fig. 1. Evolution of ultrafast laser systems. 


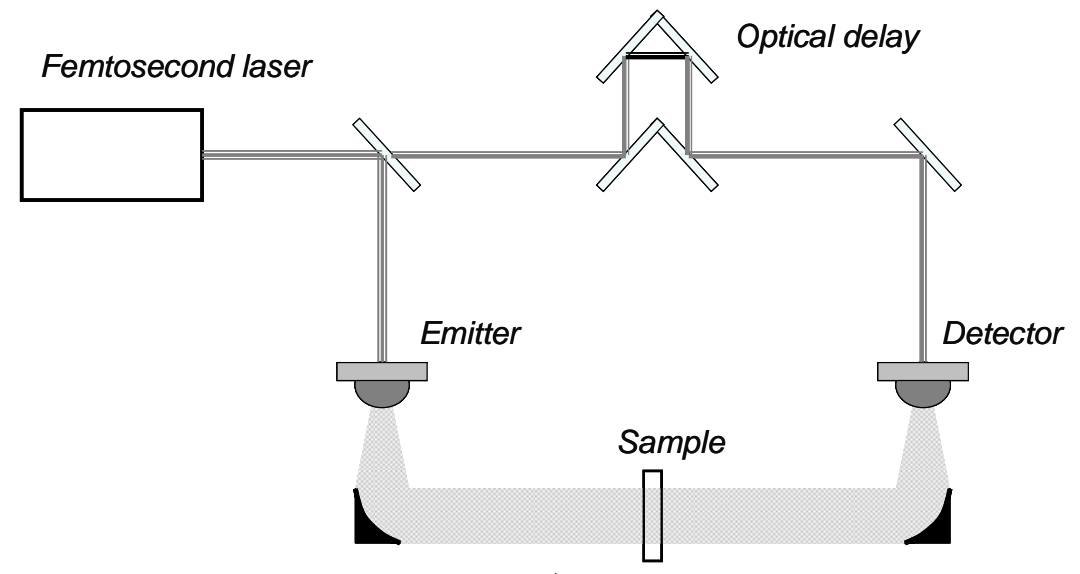

a)

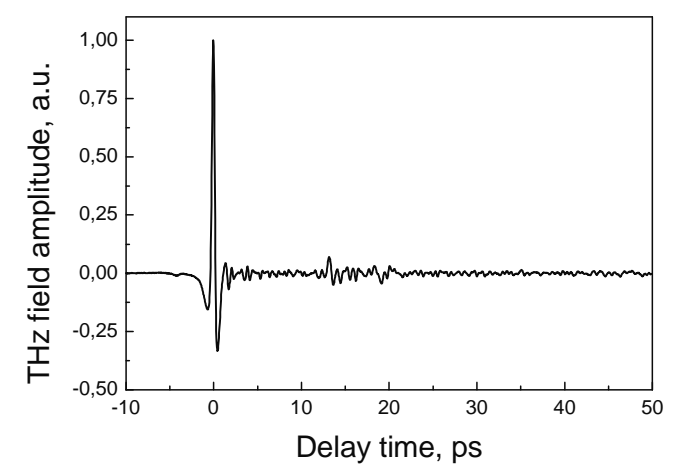

b)

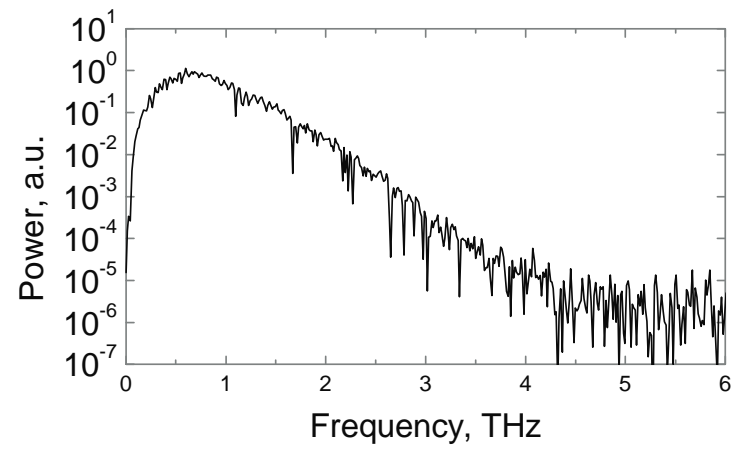

c)

Fig. 2. THz-TDS system (a), typical temporal shape of THz pulse (b), and its Fourier spectrum (c). 


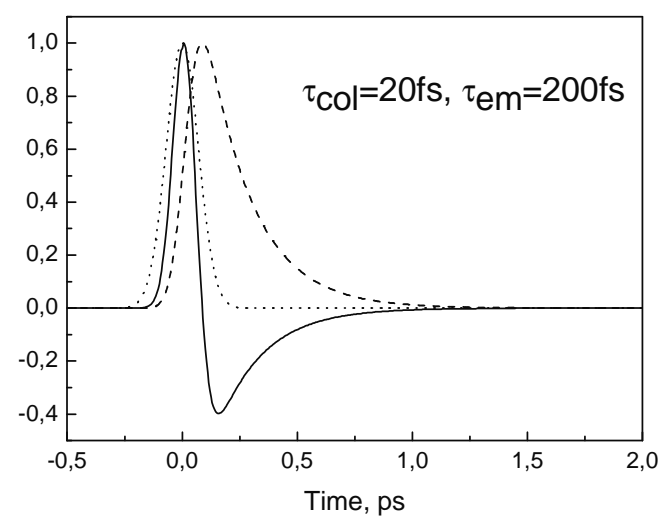

a)

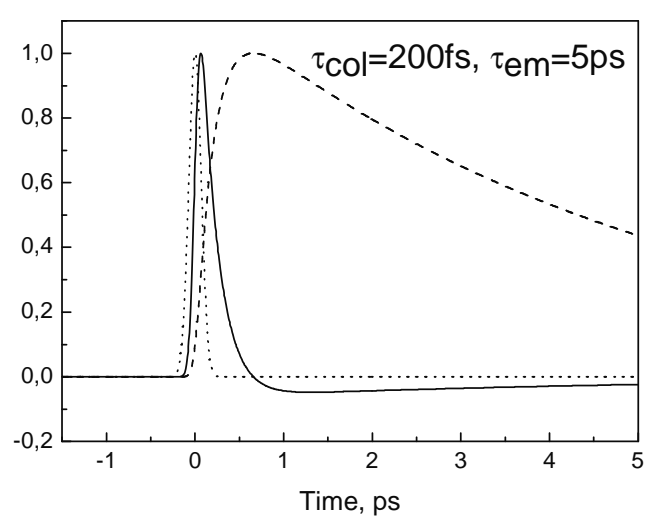

b)

Fig.3. Photocurrent transients (dashed lines) and THz pulses (full lines) emitted by devices made from semiconductors with different electron lifetimes $\left(\tau_{\mathrm{em}}\right)$ and their collision times $\left(\tau_{\mathrm{col}}\right)$. Time profile of the optical pulse is shown by dotted lines; the optical pulse duration was $100 \mathrm{fs}$. 


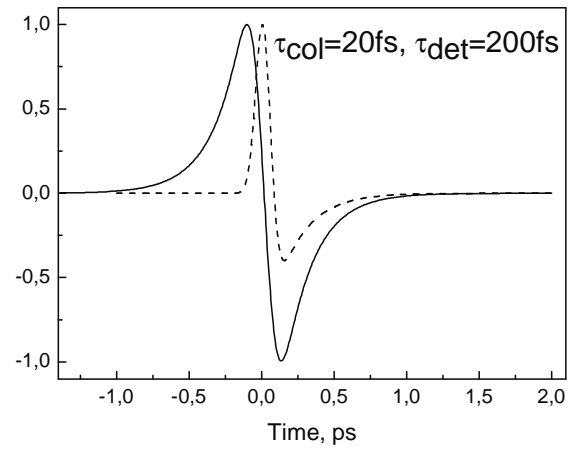

a)

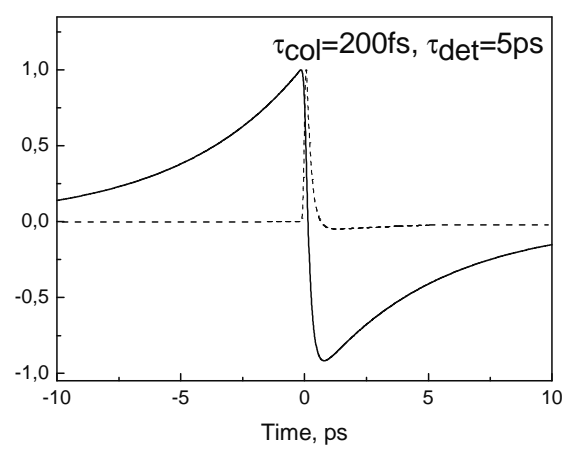

b)

Fig. 4. THz pulses (dashed lines) and transients measured by the detectors (full lines) made from two different materials with electron lifetimes $\left(\tau_{\mathrm{det}}\right)$ and their collision times $\left(\tau_{\mathrm{col}}\right)$ indicated on the graphs; the optical pulse duration was $100 \mathrm{fs}$. 


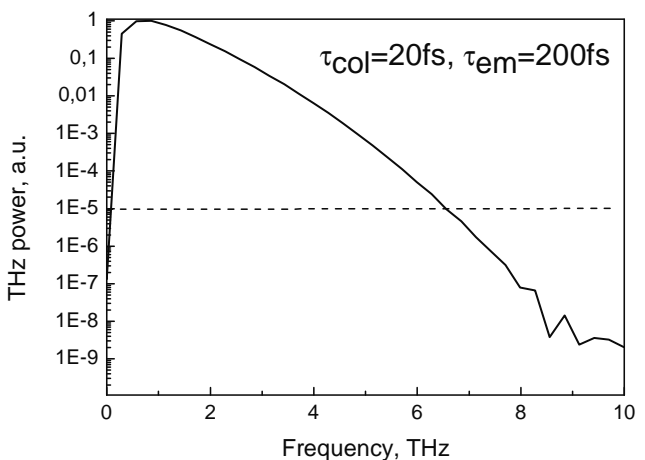

a)

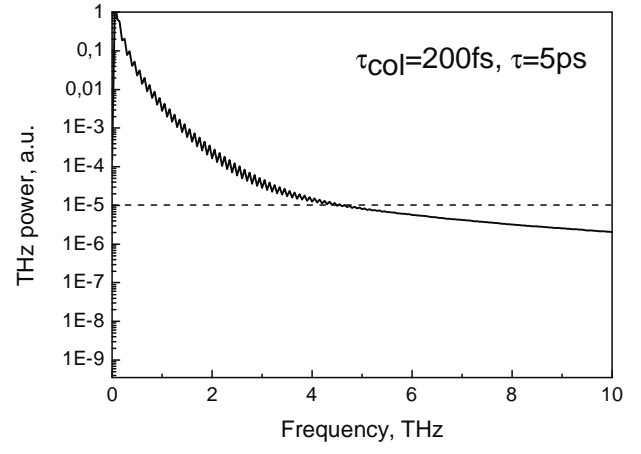

b)

Fig. 5. Fourier spectra of a THz-TDS system with emitter and detector made of LTG GaAs (a) and SI GaAs (b). 


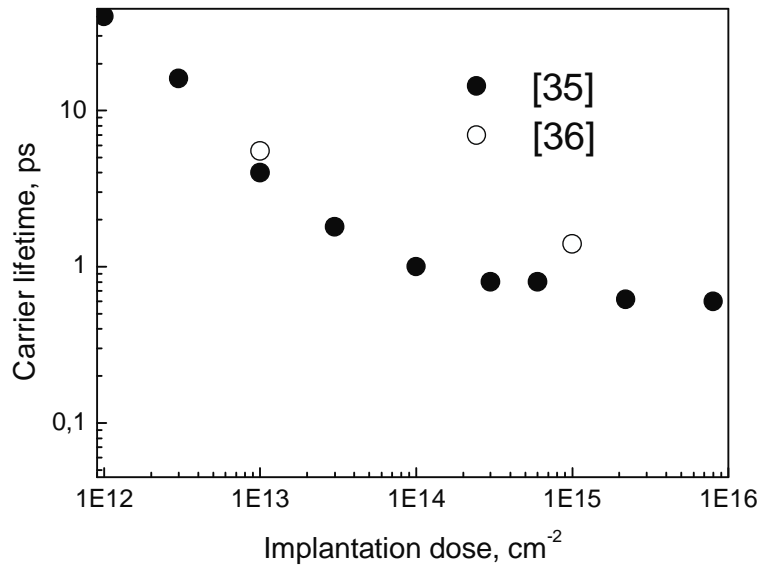

Fig. 6. Carrier lifetime of radiation-damaged $\mathrm{SOS}$ as a function of $\mathrm{O}^{+}$-implantation dose measured by optical pump - optical probe [35] and optical pump -THz probe [36] techniques. 


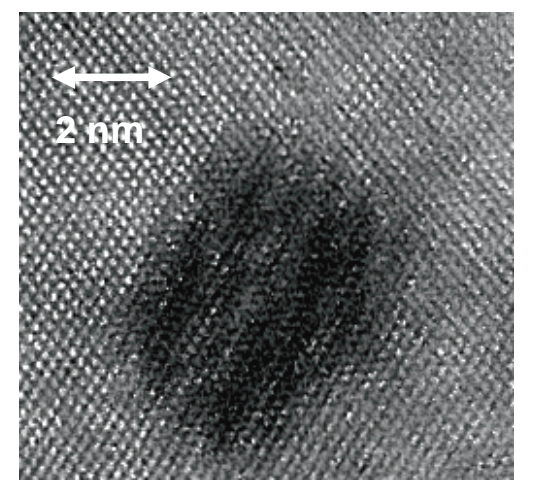

Fig. 7. High-resolution TEM picture of an As-precipitate in LTG GaAs, grown at $250^{\circ} \mathrm{C}$ and annealed at $600^{\circ} \mathrm{C}$. 


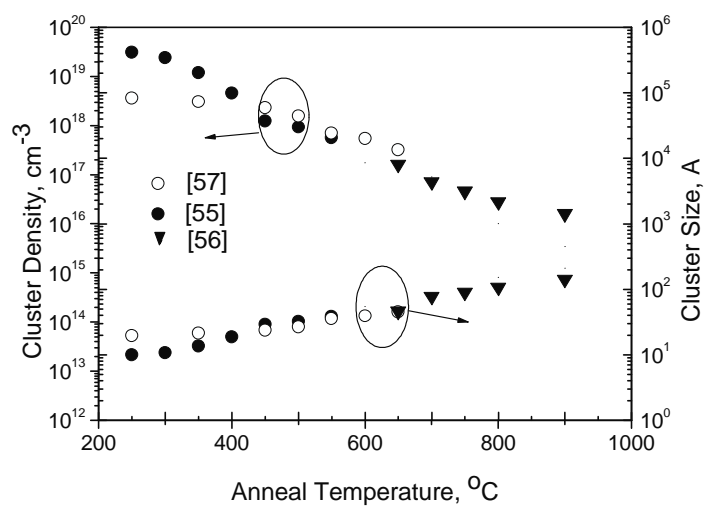

Fig. 8. As-cluster density and their size as functions of the anneal temperature. 

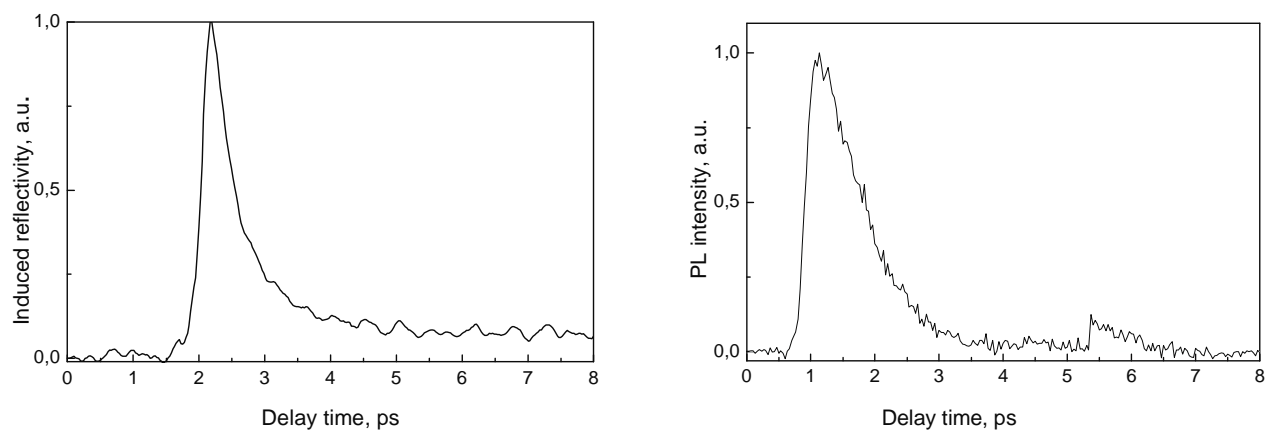

a)

b)

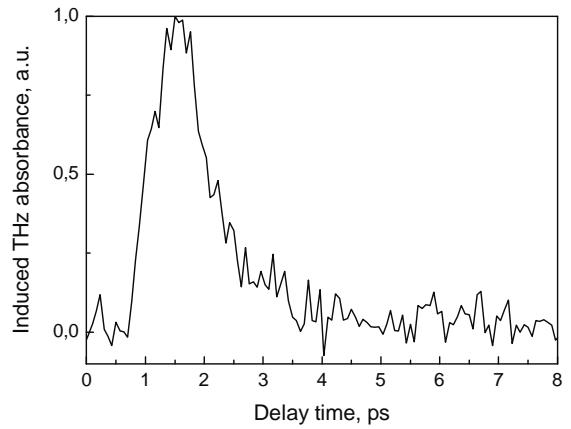

c)

Fig. 9. Results of three different experiments: transient reflectance (a), photoluminescence (b), and optical pump-THz probe measurement (c) performed on the same annealed LTG GaAs layer [76]. 


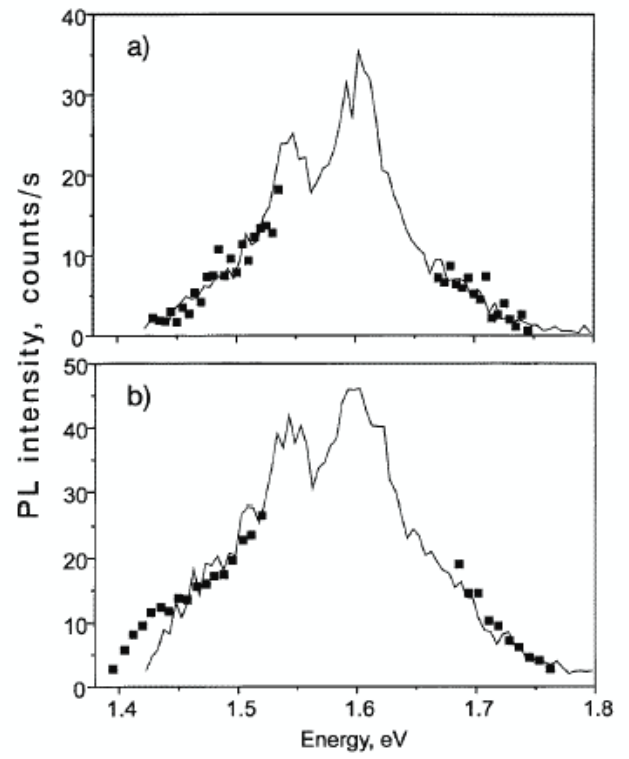

Fig. 10. PL spectra in As-ion implanted (a) and as-grown LTG (b) GaAs. Points are the experimental data measured at the peaks of PL transients and full curves are the results of a Monte Carlo molecular dynamics simulation for carrier trapping times of $30 \mathrm{fs}$ ( a) and $70 \mathrm{fs}$ ( b) [70]. 


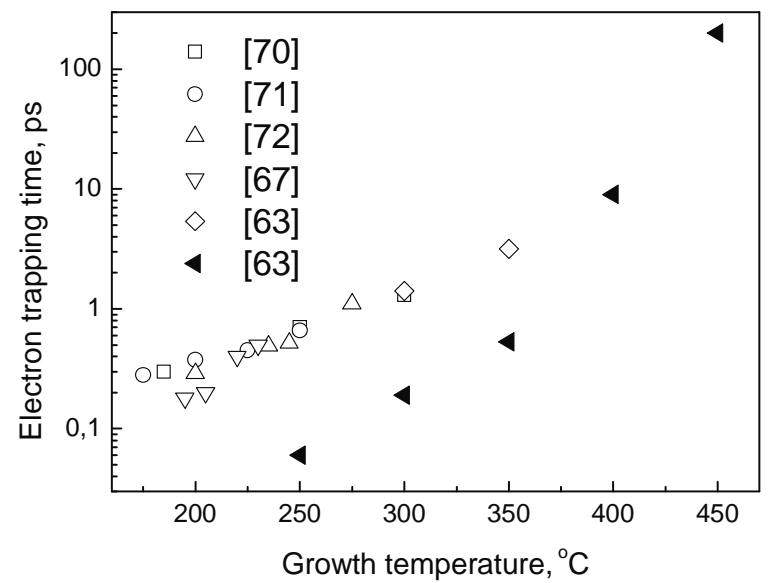

Fig. 11. Electron trapping time in as-grown (full symbols) and annealed at $600^{\circ} \mathrm{C}$ (empty symbols) LTG GaAs as a function of the growth temperature. 


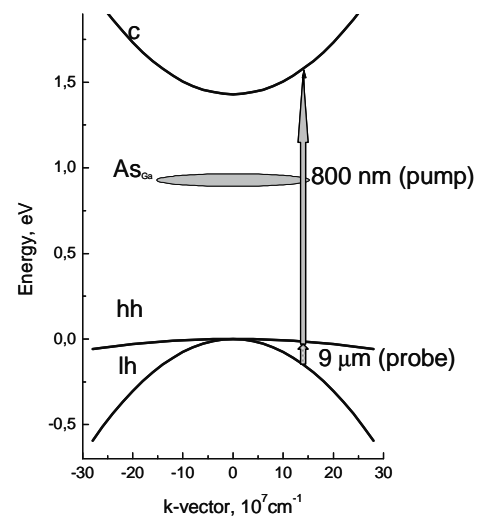

Fig. 12. Band structure of LTG GaAs with indicated electron transitions corresponding to the optical pump - mid-infrared probe experiment [79]. 


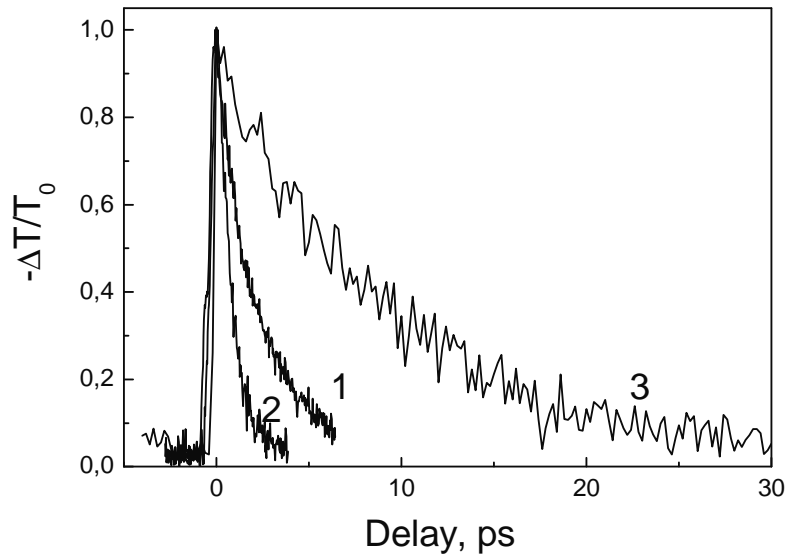

Fig. 13. Optically induced mid-infrared probe transmittance transients measured on undoped (1), Sidoped (2), and Be-doped, as-grown LTG GaAs layers [79]. 


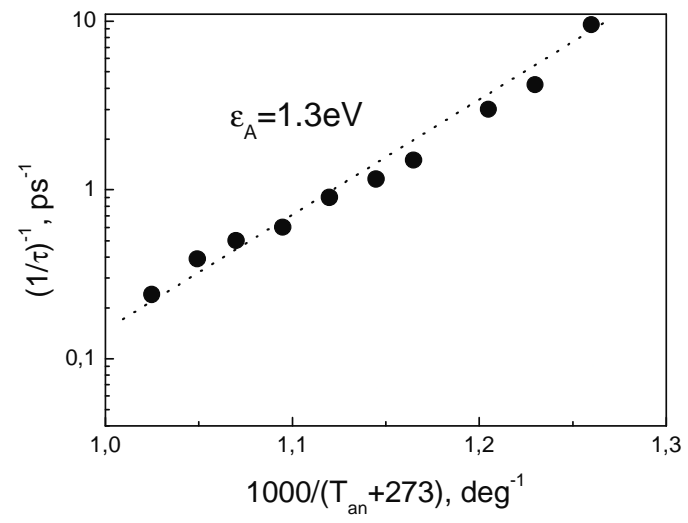

Fig. 14. Arrhenius plot of the inverse electron trapping time in As-implanted annealed GaAs layers [42]. 


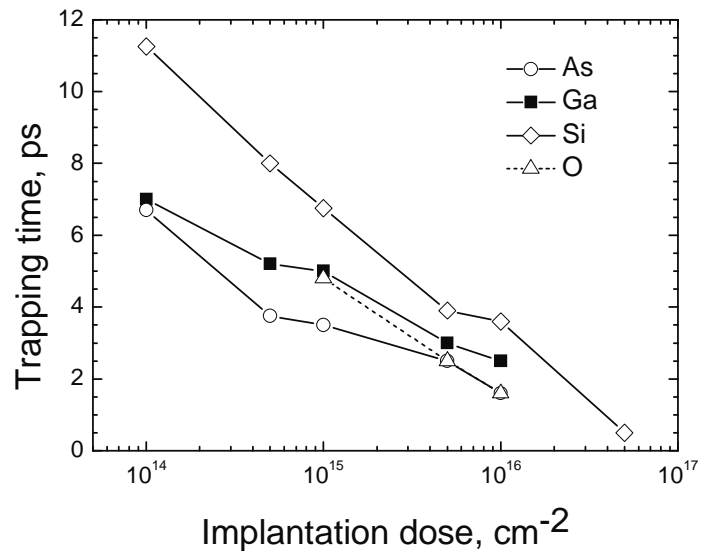

Fig. 15. Electron trapping time in GaAs as a function of the dose of four different implants. Ion energy was $2 \mathrm{MeV}\left(\mathrm{As}, \mathrm{Ga}\right.$ and $\mathrm{O}$ ) and $1 \mathrm{MeV}(\mathrm{Si})$; all samples were annealed at $600^{\circ} \mathrm{C}$ [88]. 


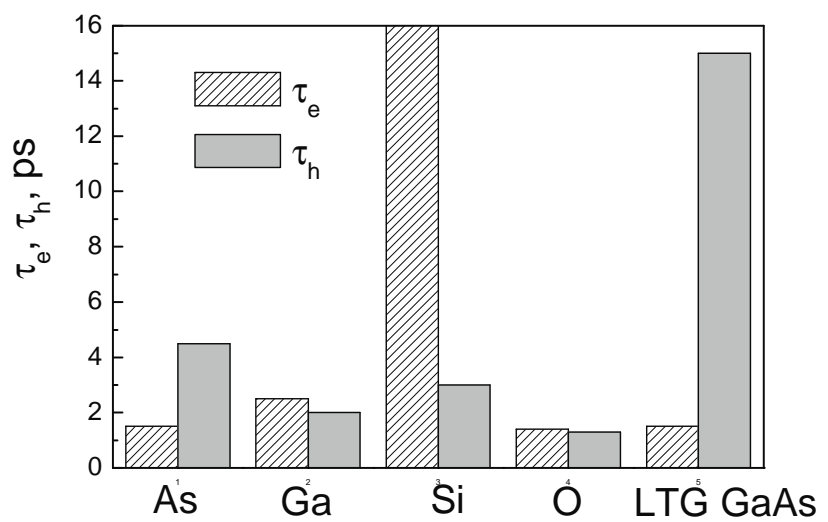

Fig. 16. Comparison of the electron and hole trapping times in various non-stoichiometric GaAs crystals [94]. 


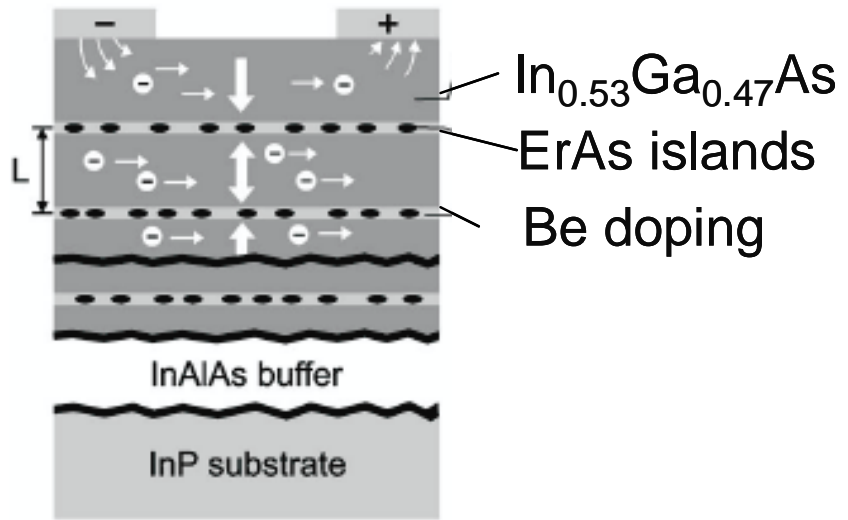

Fig. 17. Vertical cross-section of the ErAs/InGaAs supperlattice. Reprinted from [112] copyright 2008 American Institute of Physics. 


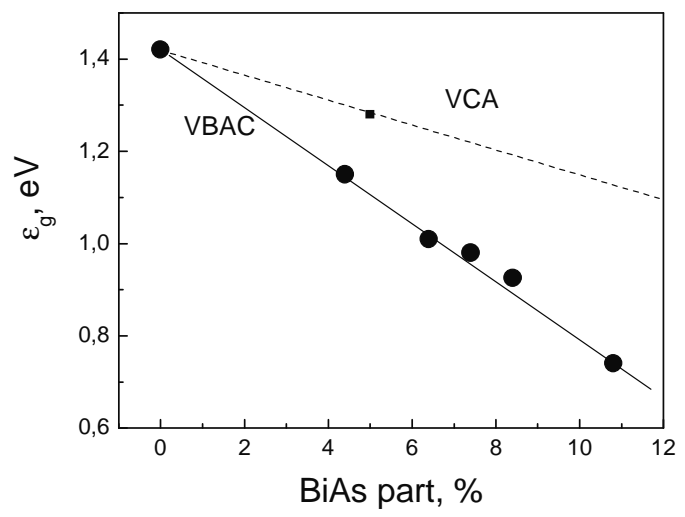

Fig. 18. Energy bandgap dependence on the BiAs content in GaBiAs alloy. Predictions of the virtual crystal approximation (VCA) and valence band anti-crossing (VBAC) model are shown by straight lines [123]. 


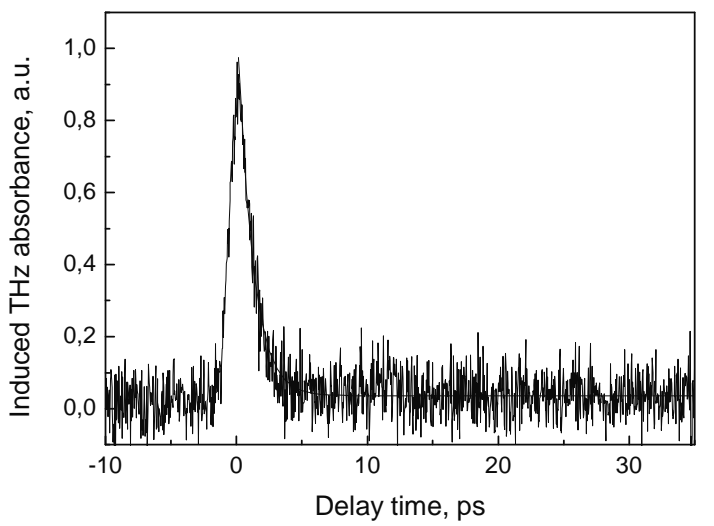

Fig. 19. Results of the $1 \mu \mathrm{m}$ pump - THz probe measurement on GaBiAs layer. 


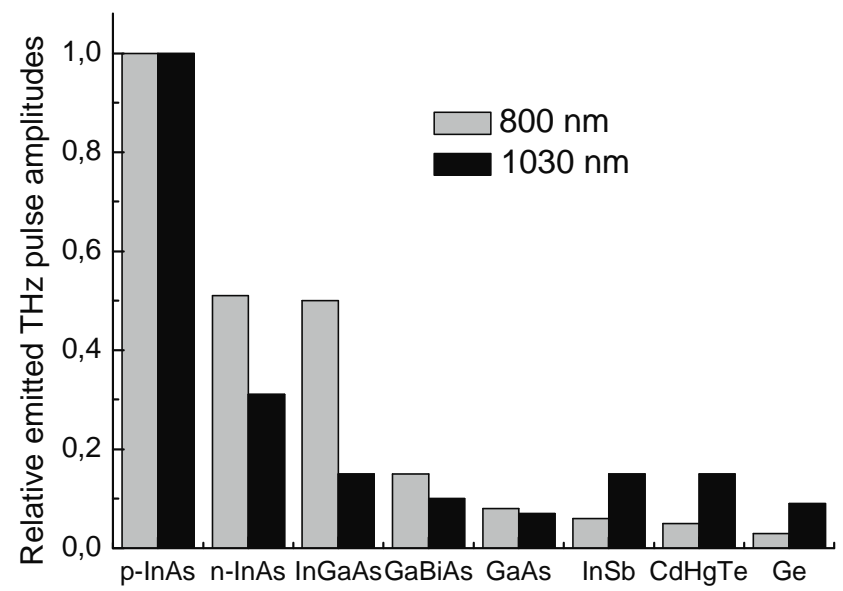

Fig. 21. Comparison of $\mathrm{THz}$ amplitudes emitted from the surfaces of several semiconductors after their excitation by femtosecond optical pulses of two different wavelengths. 
Table I. Dependences of THz pulse amplitude of two polarizations radiated due to non-linear optical effects from different planes of zinc-blende crystals. Coefficients at various trigonometric functions depend on material parameters and on the optical pulse incident angle.

\begin{tabular}{|l|l|l|}
\hline $\begin{array}{c}\text { Crystal } \\
\text { face }\end{array}$ & \multicolumn{1}{|c|}{ OR } & \multicolumn{1}{|c|}{ EFIOR } \\
\hline $\begin{array}{l}\text { (111) } \\
\text { P-polar. } \\
\text { S-polar. }\end{array}$ & $\sim\left(a_{111}+c_{111} \cos 3 \phi\right)$ & $\sim\left(A_{111}+C_{111} \cos 3 \phi\right)$ \\
(100) & $\sin 3 \phi$ & $\sim \sin 3 \phi$ \\
P-polar. & $\sim \cos 2 \phi$ & $\sim A_{100}$ \\
S-polar. & $\sim \sin 2 \phi$ & 0 \\
\hline $\begin{array}{l}\text { (110) } \\
\text { P-polar. }\end{array}$ & $\sim\left(b_{110} \sin \phi+c_{110} \sin 3 \phi\right)$ & $\sim\left(A_{110}+B_{110} \cos 2 \phi\right)$ \\
S-polar. & $\sim\left(d_{110} \cos \phi+e_{110} \cos 3 \phi\right)$ & $\sim \sin 2 \phi$ \\
\hline $\begin{array}{l}\text { (112) } \\
\text { P-polar. }\end{array}$ & $\sim\left(a_{112}+b_{112} \cos \phi+c_{112} \cos 2 \phi+d_{112} \cos 3 \phi\right)$ & $\sim\left(A_{112}+B_{112} \cos \phi+C_{112} \cos 2 \phi+D_{112} \cos 3 \phi\right)$ \\
S-polar. & $\sim\left(e_{112} \sin \phi+f_{112} \sin 2 \phi+g_{112} \sin 3 \phi\right)$ & $\sim\left(E_{112} \sin \phi+F_{112} \sin 2 \phi+G_{112} \sin 3 \phi\right)$ \\
\hline
\end{tabular}




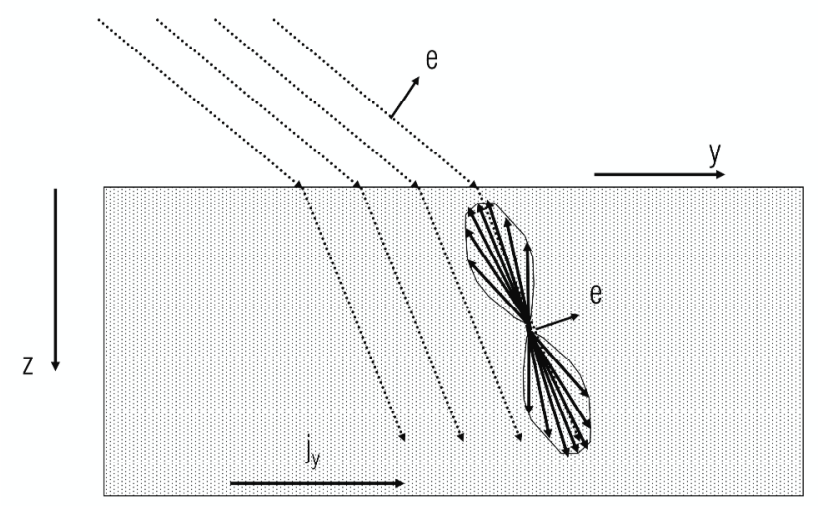

Fig. 21. Illustration of the surface photocurrent appearing due to the optical alignment of the electrons and their diffusive scattering at the surface. 


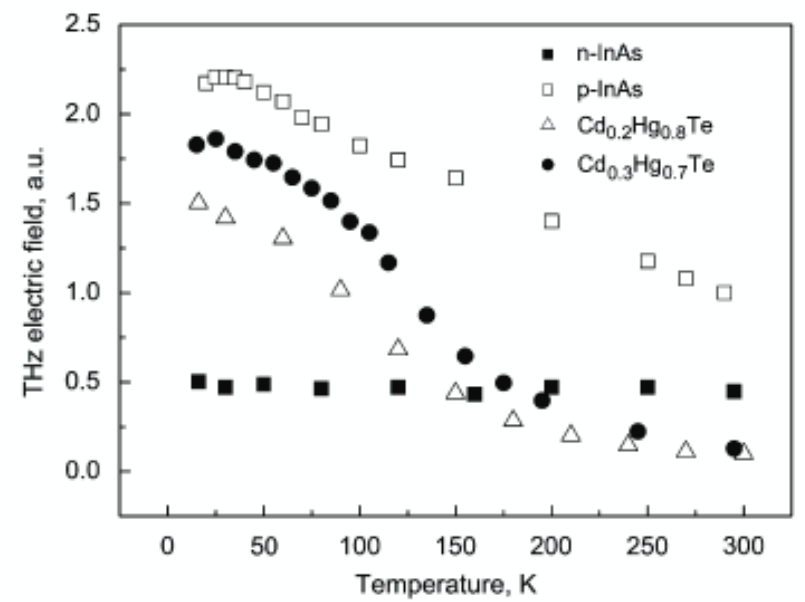

Fig. 22. THz electrical field, radiated from various semiconductor surfaces, amplitude dependence on the temperature [155]. 


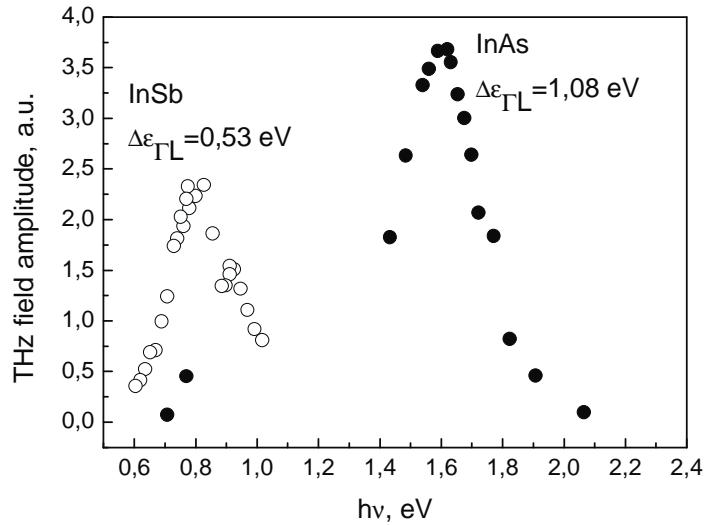

Fig. 23. Radiated THz pulse amplitudes as functions of the photon energy for two narrow-gap semiconductors [156]. Intervalley energy separation for InSb and InAs determined from the shape of these graphs are also indicated on the Figure. 


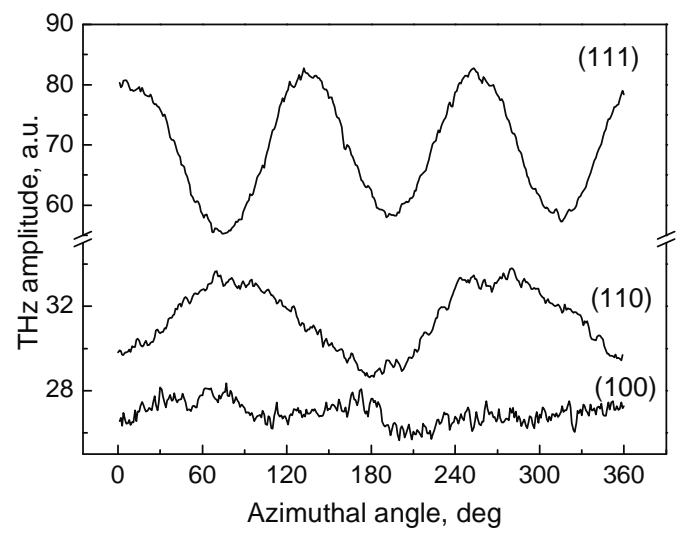

Fig. 24. The azimuthal angle dependences of $\mathrm{THz}$ signal amplitude measured by photoexciting three different planes of $n$-type Ge crystals [164]. 